ARTICLE

\title{
Glycogen synthase kinase $3 \beta$ ubiquitination by TRAF6 regulates TLR3-mediated pro-inflammatory cytokine production
}

Ryeojin Ko${ }^{1}$, Jin Hee Park ${ }^{1}$, Hyunil Ha², Yongwon $\mathrm{Choi}^{2}$ \& Soo Young Lee ${ }^{1}$

TRAF6 is critical for the production of inflammatory cytokines in various TLR-mediated signalling pathways. However, it is poorly understood how TRAF6 regulates TLR3 responses. Here we demonstrate that GSK3 $\beta$ interacts with TRAF6 and positively regulates the TLR3-mediated signalling. Suppression of GSK3 $\beta$ expression or its kinase activity drastically reduces the production of inflammatory cytokines and the induction of c-Fos by decreasing ERK and p38 phosphorylation. GSK3 $\beta$ physically associates with TRAF6 in a TLR3 ligand poly I:C-dependent manner. TRAF6 is determined to be a direct E3 ligase for GSK3 $\beta$, and TRAF6-mediated GSK3 $\beta$ ubiquitination is essential for poly I:C-dependent cytokine production by promoting the TLR3 adaptor protein TRIF-assembled signalling complex.

\footnotetext{
${ }^{1}$ Department of Life Science and the Research Center for Cellular Homeostasis, Ewha Womans University, Seoul 120-750, South Korea. ${ }^{2}$ Department of Pathology and Laboratory Medicine, University of Pennsylvania Perelman School of Medicine, Philadelphia, Pennsylvania 19104, USA. Correspondence and requests for materials should be addressed to S.Y.L. (email: leesy@ewha.ac.kr).
} 
T oll-like receptors (TLRs) comprise a class of conserved type I transmembrane pattern recognition receptors ${ }^{1}$ that recognize pathogen-associated molecular patterns and play a critical role in the host cell defence against microbial pathogens ${ }^{2}$. The recognition of pathogen-associated molecular patterns by TLRs activates multiple pathways that mediate immune responses to produce immune mediators, including pro-inflammatory cytokines, chemokines and type I interferons $(\text { IFNs })^{2-4}$. In particular, TLR3 signalling through the recognition of double-stranded RNA is crucial for antiviral responses ${ }^{5-7}$. Upon ligand binding to TLR3, the sole cytoplasmic adaptor molecule toll-interleukin 1 receptor homology-domaincontaining adapter-inducing interferon- $\beta$ (TRIF) is recruited to the TLR signalling complex ${ }^{8,9}$. The TLR3-TRIF signalling complex further triggers the recruitment of downstream signalling molecules, including tumour necrosis factor (TNF)

a
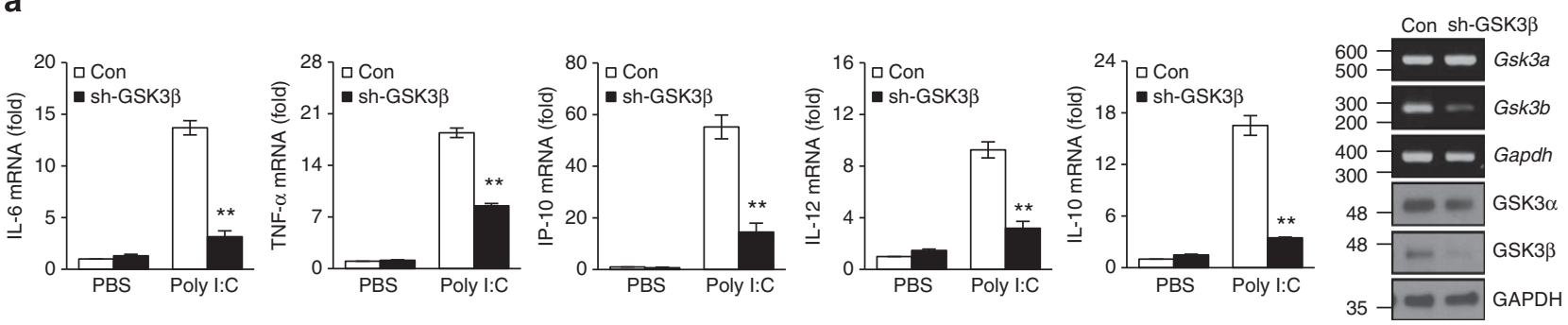

b

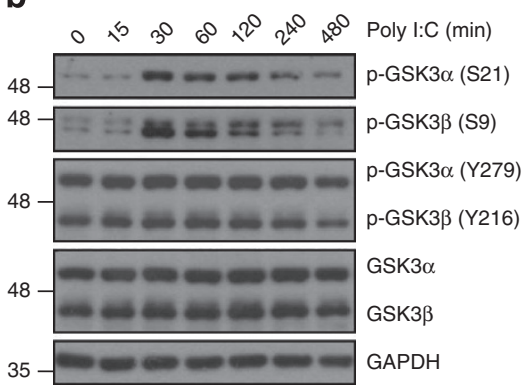

C

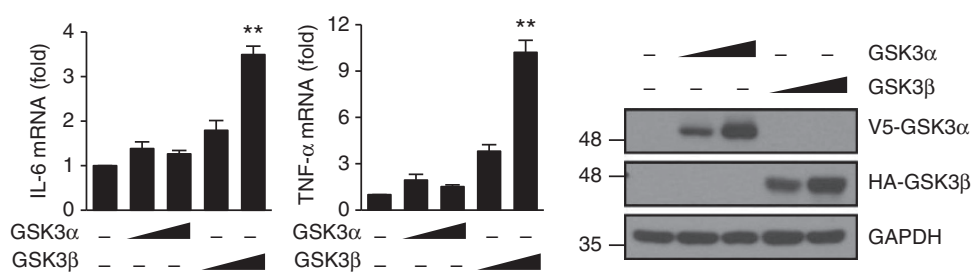

d
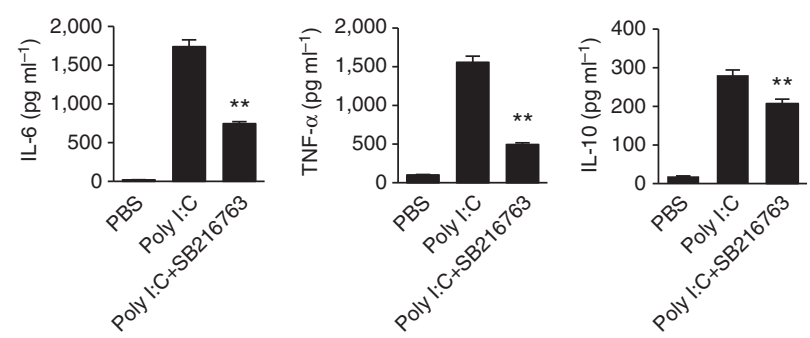

e
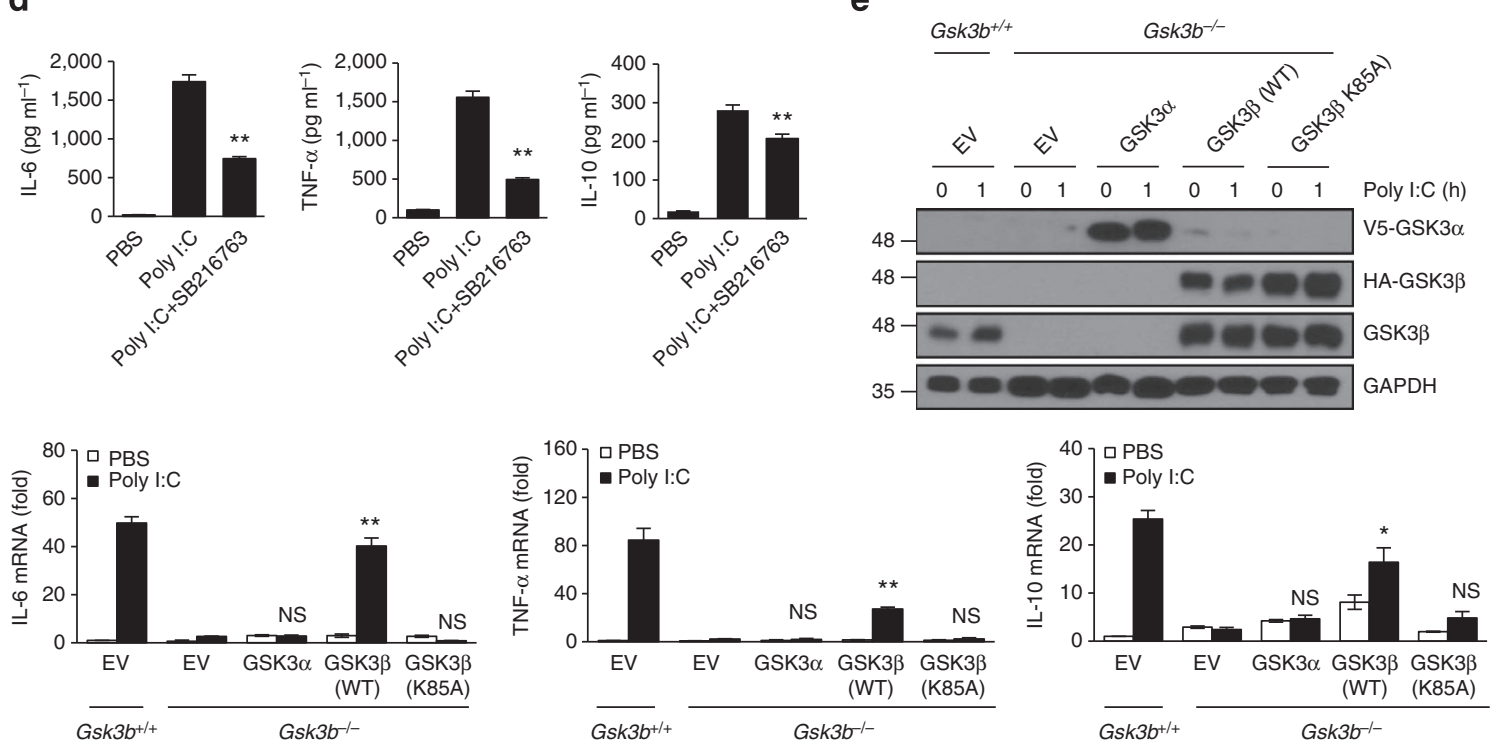

Figure 1 | GSK3 $\beta$ but not GSK3 $\alpha$ is involved in TLR3-mediated pro-inflammatory cytokine production. (a) RAW264.7 cells stably expressing control shRNA (Con) or GSK3 $\beta$-specific shRNA (sh-GSK3 $\beta$ ) were stimulated with $10 \mu \mathrm{g} \mathrm{ml}{ }^{-1}$ poly I:C for $1 \mathrm{~h}$. Levels of IL-6, TNF- $\alpha$, IP-10, IL-12 and IL-10 mRNA were determined by real-time PCR analysis, and the values were normalized to $\beta$-actin mRNA expression. GSK3 $\beta$ knockdown was confirmed by reverse transcription-PCR and western blotting. (b) BMDMs were treated with $10 \mu \mathrm{g} \mathrm{ml}-1$ poly I:C for the indicated time points. Whole-cell lysates were immunoblotted with antibodies to the molecules along the right margin. (c) HEK293-TLR3 cells were transiently transfected with V5-GSK3 $\alpha$ or HA-GSK3 $\beta$ plasmids. Levels of IL- 6 and TNF- $\alpha$ mRNA were determined as described in $\mathbf{a}$. Expression of the transduced proteins was detected by western blotting with anti-V5 (for GSK3 $\alpha$ ) and anti-HA (for GSK3 3 ). (d) BMDMs were preincubated for $1 \mathrm{~h}$ with or without $10 \mu \mathrm{M}$ SB216763 and stimulated with or without $10 \mu \mathrm{g} \mathrm{ml}^{-1}$ poly I:C for $20 \mathrm{~h}$. Levels of IL-6, TNF- $\alpha$ and IL-10 in culture supernatants were determined by enzyme-linked immunosorbent assay. (e) Gsk3b-/- MEFs were transiently transfected with V5-GSK3 $\alpha$, HA-GSK3 $\beta$ (WT) or GSK3 $\beta$ (K85A) plasmids, and cells were stimulated with $10 \mu \mathrm{g} \mathrm{ml}^{-1}$ poly I:C for $1 \mathrm{~h}$. Levels of IL-6, TNF- $\alpha$ and IL-10 mRNA were determined as described in $\mathbf{a}$. Expression of the transduced proteins was detected by western blotting as described in c. Data are presented as the mean \pm s.d. from at least three independent experiments. Statistical analyses were calculated using the Student's $t$-test $\left({ }^{\star} P<0.05 ;{ }^{*} P<0.01 ; N S\right.$, not significant). 
receptor-associated factor 3 (TRAF3) $^{10}$, TRAF6 (ref. 11) and receptor-interacting protein 1 (RIP1) $)^{12}$, which lead to the activation of IFN regulatory factor 3 (IRF3) ${ }^{13}$, activator protein $1(\mathrm{AP} 1)^{14}$ and nuclear factor-kappa $\mathrm{B}(\mathrm{NF}-\kappa \mathrm{B})^{15}$. While the TLR3-mediated signalling pathways in type I IFN production have been well explored, little is known about their regulatory mechanisms in pro-inflammatory cytokine production.

Glycogen synthase kinase 3 (GSK3) is a highly conserved serine/threonine kinase that was originally identified as a regulator of glycogen metabolism ${ }^{16}$. Two highly related isoforms of GSK3 exist, GSK3 $\alpha$ and GSK3 $\beta$, and they are ubiquitously expressed in mammalian tissues ${ }^{17}$. Although both isoforms share similar structural features, they are not functionally identical ${ }^{18}$. GSK3 $\beta$ plays crucial roles in various signal pathways that regulate multiple cellular functions, including metabolism, cell proliferation, differentiation and development ${ }^{19-21}$. GSK3 $\beta$ is also involved in diverse TLR signalling ${ }^{22,23}$. For example, GSK3 $\beta$ has been identified as a key mediator of pro-inflammatory cytokine production, including interleukin (IL)-6, TNF- $\alpha$, IL-12p40, IL-1 $\beta$ and IFN- $\gamma$, and anti-inflammatory cytokine IL-10 production by regulating CREB activity in Myd88-dependent TLR pathways ${ }^{24,25}$. In addition, GSK3 $\beta$ differentially regulates the production of lipopolysaccharide (LPS)-induced IL-I $\beta$ and the endogenous IL-1 receptor antagonist through ERK activity ${ }^{26}$. Another study demonstrated that GSK3 $\beta$ regulated IFN- $\gamma$-induced signal transducer and activator of transcription 3 (STAT3) activity and was required for the synergistic action of LPS and IFN- $\gamma$ on IL-6 cytokine production ${ }^{27}$. Although these studies clearly document the importance of GSK3 $\beta$ in TLR-mediated cytokine production, little is known about the role of GSK3 $\beta$ in TLR3 signalling.

In this report, we show that GSK3 $\beta$ is essential for TLR3-mediated ERK and p38 activation, c-Fos induction and pro-inflammatory cytokine production. We also find that GSK3 $\beta$ undergoes a lysine (K)-63 chain ubiquitination, which is important for assembling the TRIF signalling complex for TLR3 signalling. Our findings provide insights into the molecular mechanisms underlying the regulatory function of GSK3 $\beta$ in TLR3-mediated pro-inflammatory cytokine production.

\section{Results}

GSK3 $\beta$ regulates TLR3-triggered innate immune response. Previous reports demonstrated the crucial roles of GSK3 $\beta$ in TLR-mediated pro-inflammatory cytokine production through the myeloid differentiation factor 88 (MyD88)-dependent pathway $24,25,28,29$. However, how GSK3 $\beta$ regulates TLR3 signalling through a TRIF-dependent pathway ${ }^{8,9,30}$ remains unclear. To examine the involvement of GSK3 $\beta$ in TLR3 signalling, we generated the RAW264.7 macrophage cell line stably expressing a GSK3 $\beta$-specific short hairpin RNA (shRNA) (Fig. 1a). In real-time PCR analysis, the messenger RNA (mRNA) levels of pro-inflammatory cytokines, including IL-6, TNF- $\alpha$, interferon- $\gamma$-inducible protein 10 (IP-10) and IL-12, greatly decreased in the GSK3 $\beta$ knockdown RAW264.7 cells compared with the levels in control cells after a TLR3 ligand, poly I:C, stimulation (Fig. 1a). In parallel with suppression of mRNAs, knockdown of GSK3 $\beta$ led to a decrease TLR3-mediated IL-6 and TNF- $\alpha$ protein production (Supplementary Fig. 1a). The differential effects of GSK3 $\beta$ inhibition on production of proand anti-inflammatory cytokines after TLR2, TLR4, TLR5 and TLR9 stimulation have been reported ${ }^{24}$. Unlike those TLRs, TLR3 stimulation in GSK3 $\beta$ knockdown RAW264.7 cells showed a decrease in anti-inflammatory cytokine IL-10 production in mRNA and protein levels (Fig. 1a; Supplementary Fig. 1b).
Although both GSK3 $\alpha$ and GSK3 $\beta$ were phosphorylated in response to poly I:C (Fig. 1b), overexpression of GSK3 $\beta$ but not its homologue GSK $3 \alpha$ significantly elevated IL- 6 and TNF- $\alpha$ mRNA expression in a dose-dependent manner (Fig. 1c). We next used a GSK3 inhibitor SB216763 to determine whether the kinase activity of GSK3 was responsible for inflammatory cytokine production. GSK3 inhibition with SB216763 resulted in a substantial reduction in IL-6, TNF- $\alpha$ and IL-10 levels compared with the levels in untreated cells after poly I:C stimulation (Fig. 1d). Importantly, poly I:C-induced mRNA and protein expression levels of IL-6, TNF- $\alpha$ and IL-10 were impaired in $G s k 3 b^{-1-}$ mouse embryonic fibroblasts (MEFs) (Fig. 1e; Supplementary Fig. 2). Reconstitution with GSK3 $\beta$ but not GSK $3 \alpha$ or the kinase inactive GSK3 $\beta$ (K85A) mutant into $G s k 3 b^{-1}$ MEFs by transient overexpression restored the poly I:C-induced mRNA expression of IL- 6, TNF- $\alpha$ and IL-10 (Fig. 1e). Consistently, silencing of GSK3 $\beta$ but not GSK3 $\alpha$ in bone marrow-derived macrophages (BMDMs) inhibited induction of inflammatory cytokines, including IL-6, TNF- $\alpha$ and IL-10 (Supplementary Fig. $3 \mathrm{a}-\mathrm{c}$ ). Thus, these results suggest that GSK3 $\beta$ positively regulates TLR3-mediated inflammatory cytokine production and that the kinase activity of GSK3 $\beta$ is required for its role in poly I:C-induced cytokine production.

GSK3 $\beta$ is involved in retinoic acid-inducible gene 1-like receptor (RLR)-mediated antiviral response $e^{31}$. Since TLR- and RLR-mediated signalling pathways share a number of components, we examined whether GSK3 $\beta$ is also involved in poly I:C-stimulated IRF3 activation and IFN- $\beta$ induction. We found that poly I:C stimulation induced increased phosphorylation of TBK1, a kinase responsible for phosphorylating IRF3 after poly I:C stimulation, in wild-type MEFs but not $G s k 3 b^{-1-}$ MEFs (Supplementary Fig. 4a). Consistently, deficiency of GSK3 $\beta$ markedly inhibited poly I:C-induced IRF3 phosphorylation and nuclear translocation (Supplementary Fig. 4a,b) as well as dimerization of IRF3 (Supplementary Fig. 4c). Consistently, neither $G s k 3 b^{-/}-\mathrm{MEFs}$ nor GSK3 $\beta$ knockdown RAW264.7 cells showed significant induction of IFN- $\beta$ upon poly I:C stimulation (Supplementary Fig. $4 d$ ). We further confirmed that silencing of GSK3 $\beta$ but not GSK $3 \alpha$ in BMDMs inhibited IFN- $\beta$ induction significantly (Supplementary Fig. 4e). It is interesting to note that GSK3 inhibition with SB216763 did not alter poly I:C-induced IFN- $\beta$ mRNA expression (Supplementary Fig. 4f), suggesting that the effect of GSK3 $\beta$ on TLR3-mediated IFN- $\beta$ induction is independent of its kinase activity. Together, these data indicate that GSK $3 \beta$ is required for TLR3-mediated IRF3 activation and the type I IFN- $\beta$ induction.

GSK3 $\beta$ regulates TLR3-mediated ERK and $\mathrm{p} 38$ activation. The mitogen-activated protein kinases (MAPKs) and the NF- $\kappa \mathrm{B}$ signalling pathways are important for inflammatory cytokine production in TLR signalling ${ }^{32-34}$. To examine whether GSK3 $\beta$ regulates MAPKs and NF- $\kappa \mathrm{B}$ activation in TLR3 signalling, we analysed the phosphorylation levels of ERK, p38, JNK and NF- $\kappa B$ p65 in GSK3 $\beta$ knockdown RAW264.7 cells. As shown in Fig. 2a, GSK3 $\beta$ knockdown markedly decreased poly I:C-induced ERK and $\mathrm{p} 38$ phosphorylation levels, whereas there were no significant differences in the phosphorylation levels of JNK and NF- $\kappa \mathrm{B}$ p65. Similar results were observed in BMDMs silenced by short interfering RNA (siRNAs) specific for GSK3 $\beta$. Although knockdown of both forms of GSK3 appears to delay poly I:Cinduced I $\mathrm{K} B-\alpha$ degradation, silencing of GSK3 $\beta$ but not GSK3 $\alpha$ significantly reduced poly I:C-induced ERK and p38 activation (Supplementary Fig. 5). These results were further confirmed in $G s k 3 b^{-1-}$ MEFs. Phosphorylation levels of ERK and p38 but not 
a

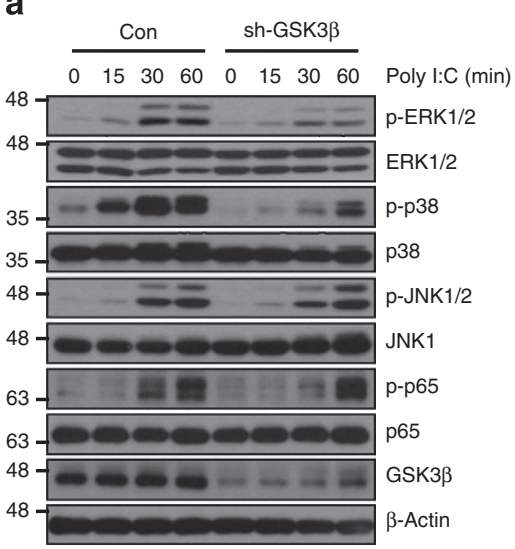

b

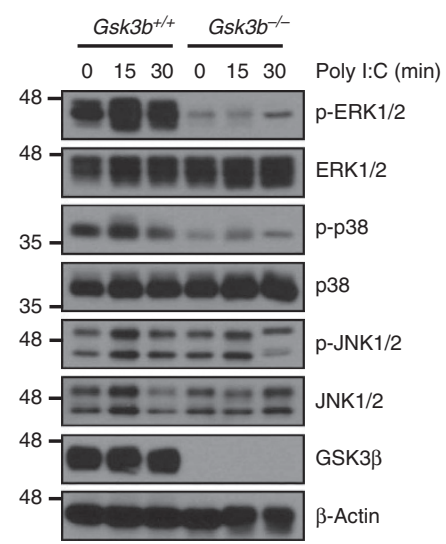

C

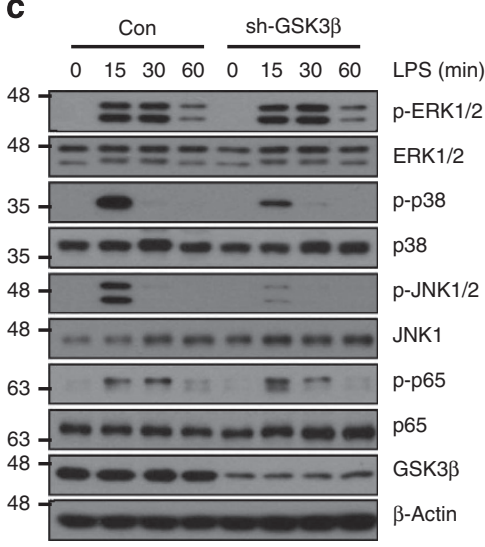

d

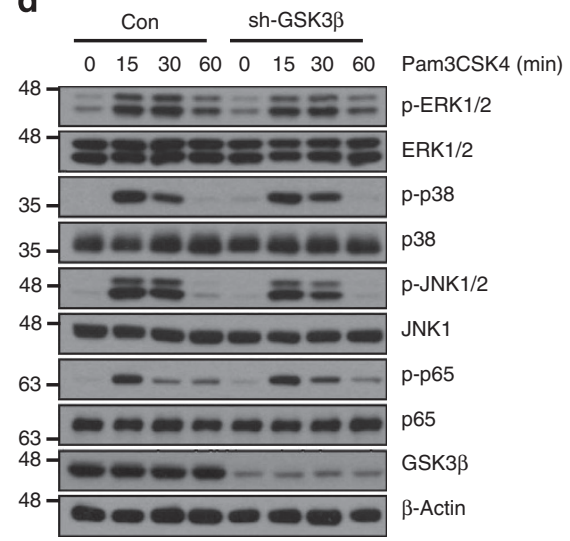

Figure 2 | GSK3 $\beta$ regulates poly I:C-mediated ERK and p38 activation. (a) Western blotting of the phosphorylation of the MAPKs ERK, p38 and JNK and

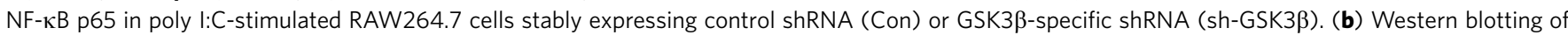
ERK, p38 and JNK phosphorylation in Gsk3b+/+ and Gsk3b-/- MEFs stimulated with $10 \mu \mathrm{g} \mathrm{ml}^{-1}$ poly I:C. (c,d) As in a, except that the cells were stimulated with $100 \mathrm{ng} \mathrm{ml}^{-1}$ LPS (c) or $100 \mathrm{ng} \mathrm{ml}^{-1}$ Pam3CSK4 (d) as indicated. Data are representative of two or three independent experiments.

JNK, IKK $\alpha / \beta, \mathrm{I} \kappa \mathrm{B}-\alpha$ and NF- $\kappa \mathrm{B}$ p65 were significantly decreased in $G s k 3 b^{-1-}$ MEFs compared with the levels in $G s k 3 b^{+1+}$ MEFs (Fig. 2b; Supplementary Fig. 6a). In reporter assays, knockdown or overexpression of GSK3 $\beta$ did not affect poly I:Cinduced NF- $\kappa B$ activation, further suggesting that GSK3 $\beta$ is not involved in TLR3-mediated NF- $\kappa \mathrm{B}$ signalling (Supplementary Fig. 6b,c). Because a TRIF-dependent pathway is also involved in TLR4 signalling ${ }^{35,36}$, we examined the effects of GSK3 $\beta$ on LPSinduced MAPKs and NF- $\kappa \mathrm{B}$ p65 phosphorylation levels. Interestingly, the GSK3 $\beta$ knockdown decreased LPS-induced phosphorylation levels of p38 and JNK but not ERK and NF- $\kappa \mathrm{B}$ p65 (Fig. 2c). In contrast, there were no significant differences between GSK3 $\beta$ knockdown RAW264.7 and control cells after a TLR2 ligand, Pam3CSK4, stimulation (Fig. 2d). It should be noted that Pam3CSK4-induced MAPKs and NF- $\kappa \mathrm{B}$ p65 phosphorylation is mediated by a Myd88-dependent, but not a TRIF-dependent, pathway. Thus, these data indicate that GSK3 $\beta$ regulates ERK and p38 MAPK activation in TRIF-dependent TLR3 signalling.

GSK3 $\beta$ regulates expression of c-Fos through ERK and p38. AP1, comprising Jun, c-Fos and ATF2, is activated in response to TLR stimulation and regulates the production of pro-inflammatory cytokines ${ }^{14,37,38}$. To identify transcription factors regulated by the TLR3-GSK3 $\beta$ axis, we separated the cytosolic and nuclear fractions of GSK3 $\beta$ knockdown RAW264.7 cells after poly I:C stimulation. Interestingly, the c-Fos protein levels in the nuclear fraction appeared drastically reduced in GSK3 $\beta$ knockdown RAW264.7 cells compared with the levels in control cells, whereas nuclear ATF2, c-Jun and NF- $\kappa \mathrm{B}$ p65 protein levels were comparable in both cell types (Fig. 3a; Supplementary Fig. 7), suggesting that GSK $3 \beta$ regulates TLR3-mediated c-Fos expression but not ATF2, c-Jun and NF- $\kappa \mathrm{B}$ p65. Consistently, treatment with the GSK3 inhibitor SB216763 or silencing of GSK3 $\beta$ but not GSK3 $\alpha$ significantly reduced c-Fos mRNA or protein after TLR3 stimulation (Fig. 3b,c), whereas overexpression of GSK3 $\beta$ but not GSK $3 \alpha$ produced a substantial increase in c-Fos mRNAs (Supplementary Fig. 8). Reconstitution of GSK3 $\beta$ but not GSK3 $\alpha$ or the kinase inactive GSK3 $\beta$ (K85A) mutant into Gsk $3 b^{-1-}$ MEFs by transient transfection restored poly I:C-induced c-Fos mRNA expression (Fig. 3d). Moreover, the knockdown of c-Fos markedly reduced poly I:C-induced IL-6 and TNF- $\alpha$ expression (Supplementary Fig. 9), confirming the importance of GSK3 $\beta$-mediated c-Fos expression in TLR3 signalling.

Considering the defects in ERK and p38 MAPK signalling in GSK3 $\beta$ knockdown RAW264.7 cells and Gsk3b ${ }^{-9}$ MEFs (Fig. 2a,b), it is likely that poly I:C-mediated ERK and p38 MAPK activation regulates c-Fos expression. To test this hypothesis, we assessed c-Fos expression in the presence of the ERK inhibitor PD98059, the p38 inhibitor SB203580, the JNK inhibitor SP600125 or the NF- $\mathrm{B}$ inhibitor BAY 11-7085. Expectedly, ERK or p38 inhibition markedly reduced poly I:C-induced c-Fos protein levels compared with the levels of the dimethylsulphoxide control (Fig. 3e,f). In contrast, nuclear c-Fos 
a

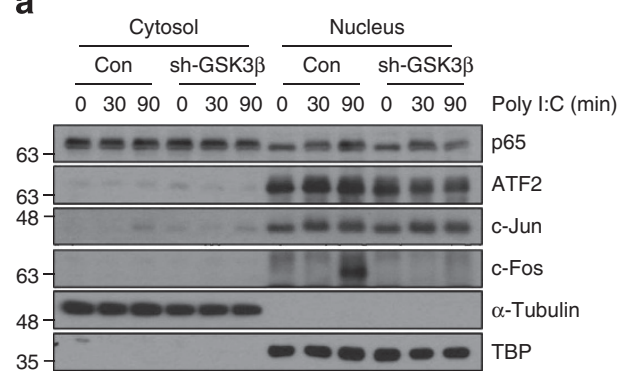

b

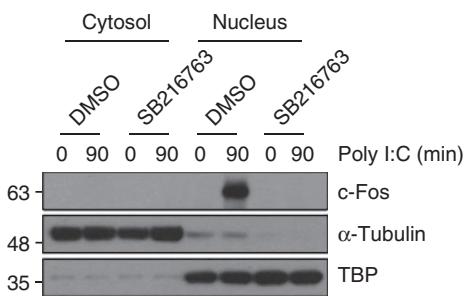

C

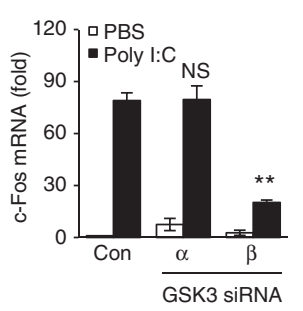

e

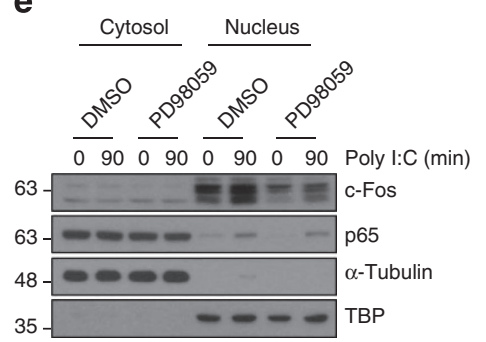

g

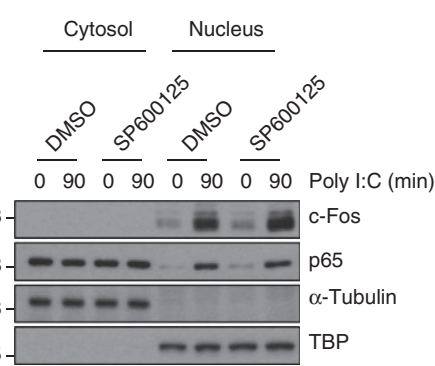

d

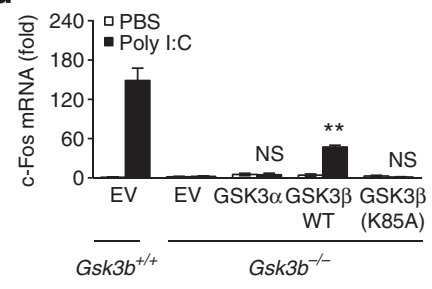

$\mathbf{f}$

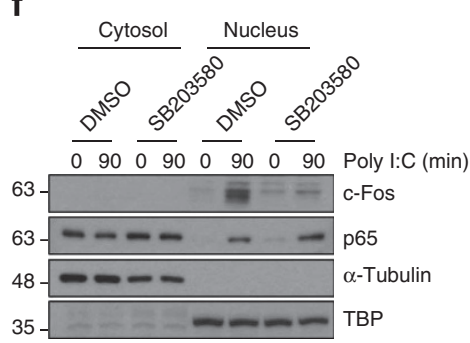

h
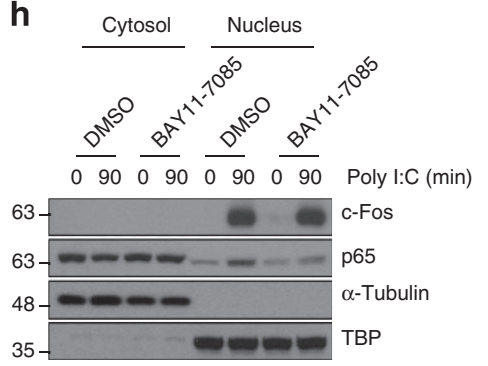

Figure 3 | GSK3 $\beta$ regulates poly I:C-mediated c-Fos induction through the ERK and p38 pathways. (a) RAW264.7 cells stably expressing control shRNA (Con) or GSK3 $\beta$-specific shRNA (sh-GSK3 $\beta$ ) were stimulated with $10 \mu \mathrm{g} \mathrm{ml} \mathrm{l}^{-1}$ poly l:C for the indicated time points. Cells were separated into cytosolic and nuclear fractions, and the protein levels of p65, ATF2, c-Jun and c-Fos were determined by western blotting. $\alpha$-tubulin and TBP served as markers for the cytosolic and nuclear fractions, respectively. (b) As in $\mathbf{a}$, except that the cells were preincubated with dimethylsulphoxide or $10 \mu \mathrm{M}$ SB216763 for $1 \mathrm{~h}$ and then stimulated with $10 \mu \mathrm{g} \mathrm{ml}^{-1}$ poly I:C for $90 \mathrm{~min}$. Cytosolic and nuclear c-Fos levels were determined by western blotting. (c) BMDMs transfected with $20 \mathrm{nM}$ control siRNAs (Con) or GSK3 $\alpha$ - or GSK3 $\beta$-specific siRNAs were stimulated with $10 \mu \mathrm{g} \mathrm{ml}^{-1}$ poly I:C for $1 \mathrm{~h}$. Levels of c-Fos mRNA were determined by real-time PCR. (d) As in c, except that Gsk3b+/+ and Gsk3b-/- MEFs were transiently transfected with V5-GSK3 $\alpha$, HA-GSK3 $\beta$ (WT) or GSK3 $\beta$ (K85A) plasmids. (e-h) As in b, except that the cells were preincubated with $10 \mu \mathrm{M}$ PD98059 (e), $10 \mu \mathrm{M}$ SB203580 (f), $25 \mu \mathrm{M}$ SP600125 (g) or $10 \mu \mathrm{M}$ BAY 11-7085 (h) for $1 \mathrm{~h}$ and then stimulated with $10 \mu \mathrm{g} \mathrm{ml}{ }^{-1}$ poly I:C for $90 \mathrm{~min}$. Cytosolic and nuclear c-Fos and p65 protein levels were determined by western blotting. Data are presented as the mean \pm s.d. from at least three independent experiments. Statistical analyses were calculated using the Student's t-test ( ${ }^{\star *} P<0.01 ; N S$, not significant).

expression was not affected by the inhibition of JNK or NF- $\mathrm{KB}$ (Fig. 3g,h). Together, these data indicate that GSK3 $\beta$ regulates poly I:C-induced c-Fos expression via ERK and p38 activation.

GSK3 $\beta$ is required for TLR3 signalling complex formation. Upon poly I:C stimulation, activated TLR3 initiates the interaction of TRIF with TRAF6, transforming growth factor $\beta$-activated kinase 1 (TAK1) and RIP1 to activate MAPKs and NF- $\kappa B$ signalling cascades ${ }^{11,12,39}$. Specifically, the TRIF-TRAF6-TAK1 axis is important for the activation of MAPKs, which in turn leads to AP1 activation ${ }^{34,39,40}$. To examine how GSK3 $\beta$ regulates MAPKs in TLR3 signalling, we first determined the roles of GSK3 $\beta$ in forming the TLR3 signalling complex following poly I:C stimulation in $G s k 3 b^{-1-}$ MEFs. In control cells, poly I:C stimulation induced recruitment of TRIF, RIP1, TRAF6, TAK1, TAK1-binding protein 1 (TAB1), TAB2 and GSK3 $\beta$ to TLR3, which persisted for at least $30 \mathrm{~min}$ (Fig. 4). Interestingly, GSK3 $\beta$ deficiency prevented the recruitment of TRAF6, TAK1, TAB1 and TAB2 to TLR3, but had no effect on the recruitment of TRIF 


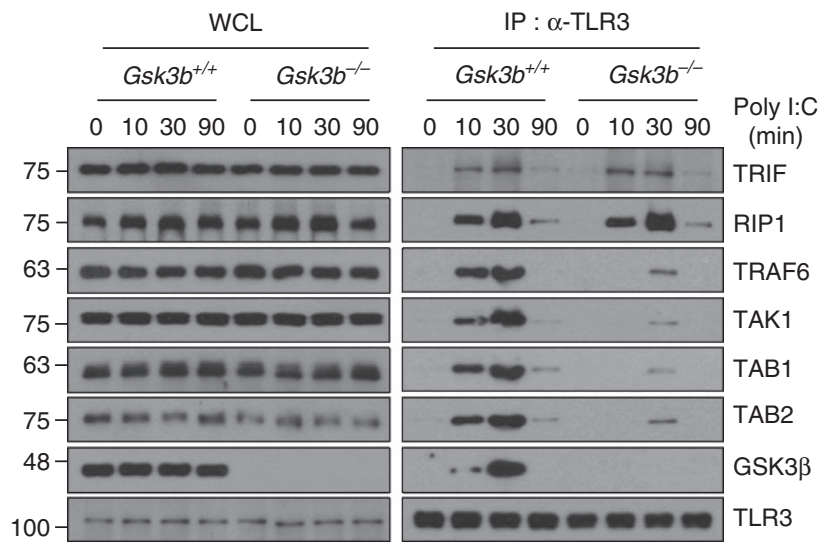

Figure 4 | GSK3 $\beta$ is required for the recruitment of the TRAF6-TAK1TAB1-TAB2 complex to TLR3. Gsk3b+/ + and Gsk3b-/- MEFs were stimulated with $10 \mu \mathrm{g} \mathrm{ml}^{-1}$ poly I:C for $10 \mathrm{~min}$ and subjected to immunoprecipitation with an anti-TLR3 antibody. TRIF, RIP1, TRAF6, TAK1, TAB1, TAB2 and GSK3 $\beta$ protein levels from whole-cell lysates $(W C L)$ and TLR3 immunocomplexes (IP: $\alpha$-TLR3) were determined by western blotting. Data are representative of two independent experiments.

and RIP1. Consistently, silencing of GSK3 $\beta$ in RAW264.7 macrophages impaired the poly I:C-triggered formation of the TLR3 signalling complex containing TRAF6, TAK1, TAB1 and TAB2 (Supplementary Fig. 10a). Notably, pretreatment with the GSK3 inhibitor SB216763 in BMDMs also blocked the formation of the poly I:C-induced TLR3 signalling complex (Supplementary Fig. 10b). Thus, these results suggest that GSK3 $\beta$ is important for recruiting the TRAF6-TAK1-TAB1-TAB2 complex to TLR3.

GSK3 $\beta$ interacts with TRAF6 and TAK1. To further explore the regulatory mechanisms of GSK3 $\beta$ in TLR3 signalling, we investigated the interaction of GSK3 $\beta$ with TRAF6 and TAK1. TAK1, a MAP3K family member ${ }^{41}$, is a critical transducer molecule downstream of TRAF6, and the TRAF6-TAK1 axis activates MAPKs in TLR signalling ${ }^{2,42,43}$. We first examined by coimmunoprecipitation experiments whether GSK3 $\beta$ associates with TRAF6 or TAK1 upon poly I:C stimulation in BMDMS. The results indicated that GSK3 $\beta$ indeed associates with TRAF6 and/or TAK1 under physiological conditions (Fig. 5a). We next examined whether TRAF6, GSK3 $\beta$ and TAK1 bind to one another, forming a ternary complex. To examine this binding, HEK293T cells were transfected with a Flag-TRAF6 construct with or without a Myc-TAK1 or HA-GSK3 $\beta$ construct. We observed that Flag-TRAF6 co-immunoprecipitated with MycTAK1 and HA-GSK3 $\beta$, indicating that TRAF6 forms a ternary complex with GSK3 $\beta$ and TAK1 (Fig. 5b). Similarly, we further confirmed the association of GSK3 $\beta$ with TRAF6 and TAK1 as well as TAK1 with TRAF6 and GSK3 $\beta$ by cotransfection studies in mammalian cells (Supplementary Fig. 11a,b). Notably, deletion of GSK3 $\beta$ prevented association of TRAF6 with TAK1 upon poly I:C stimulation (Fig. 5c). We also investigated the interaction of GSK3 $\beta$ with RIP1, an essential mediator of TLR3-induced NF- $\kappa B$ activation $^{12}$. Unlike TRAF6 and TAK1, however, RIP1 did not interact with GSK3 $\beta$ (Supplementary Fig. 12a,b), further confirming that GSK3 $\beta$ is not involved in NF- $\kappa B$ signalling mediated by the TLR3-TRIF-RIP1 axis.

TAK1 forms a complex with its adaptor proteins TAB1 and TAB2. TAB2 bridges TRAF6 to TAK1, allowing TAK1 activation $^{44-46}$. We therefore investigated whether GSK3 $\beta$ could associate with $\mathrm{TAB} 1$ or $\mathrm{TAB} 2$ and affect the formation of the signalling complex containing TRAF6, TAK1, TAB1 and TAB2. In contrast to the GSK3 $\beta$-TAK1 interaction, there was little association between GSK3 $\beta$ and TAB1 or TAB2 (Fig. 5d, lanes 3 and 4 ). However, GSK3 $\beta$ could associate with TAB1 or TAB2 in the presence of TAK1, indicating that the association of GSK3 $\beta$ with TAB1 or TAB2 requires TAK1. Notably, forced expression of GSK3 $\beta$ promotes the association of TRAF6 with the TAK1-TAB1-TAB2 complex (Fig. 5e, lane 3). Furthermore, overexpressed GSK3 $\beta$ binds to TRIF, TRAF6 and TAK1 as a complex (Fig. 5f). Altogether, these results demonstrate that GSK3 $\beta$ could form a TRIF-assembled signalling complex containing TRAF6-TAB1-TAB2-TAK1.

TRAF6 is required for ubiquitination of GSK3ק. Because TRAF6 possesses E3 ubiquitin ligase activity ${ }^{47-49}$, we next questioned whether GSK3 $\beta$ is ubiquitinated by TRAF6 in TLR3 signalling. We first identified the binding regions between TRAF6 and GSK3 $\beta$. GSK3 $\beta$ interacted with the TRAF6 (289-530) derivative containing just the coiled-coil TRAF-N domain and the conserved TRAF-C domain, whereas the $\mathrm{N}$-terminal ring and zinc-finger domains of TRAF6 spanning amino acids (aa) 1-289 failed to interact with GSK3 $\beta$ (Supplementary Fig. 13a). On the other hand, serial deletion constructs of GSK3 $\beta$ revealed that the $\mathrm{N}$-terminal region spanning aa $1-120$ is necessary for TRAF6 interaction (Supplementary Fig. 13b). We next tested whether GSK3 $\beta$ is ubiquitinated upon poly I:C stimulation in BMDMs and found that poly I:C triggered polyubiquitination of GSK3 $\beta$ (Fig. 6a). Furthermore, overexpression of TRAF6 induced GSK3 $\beta$ ubiquitination (Fig. 6b). In contrast, the catalytically inactive TRAF6 (C70A) mutant, which has lost its E3 ligase activity, lost the ability to promote GSK3 $\beta$ ubiquitination (Fig. 6c). Ubiquitination of GSK3 $\beta$ occurred mainly through K63 linkage (Supplementary Fig. 14). Unlike TRAF6, NEDD4-1 and TRAF3, two E3 ubiquitin ligases that catalyse K63 ubiquitination and function in CD40 and TLR2 signalling, respectively ${ }^{50,51}$, did not promoted GSK3 $\beta$ ubiquitination (Supplementary Fig. 15). TRAF6 deficiency markedly decreased ubiquitination of endogenous GSK3 $\beta$ compared with the ubiquitination of wild-type cells upon poly I:C stimulation (Fig. 6d). Moreover, TRAF6 induced GSK3 $\beta$ ubiquitination in vitro (Fig. 6e). These results suggest that TRAF6 is an E3 ubiquitin ligase for GSK3 $\beta$.

To search for the sites of GSK3 $\beta$ that are responsible for ubiquitination, we generated a series of full-length GSK3 $\beta$ variants containing a lysine residue mutation based on mass spectrometry (MS) analysis (Supplementary Table 1). We mutated K85, K86, K91 or K183 to arginine and then tested the susceptibility of these mutants, when expressed ectopically, to be ubiquitinated by TRAF6. TRAF6-mediated ubiquitination of GSK3 $\beta$ was substantially reduced when with K183R, but not with K85R, K86R or K91R (Fig. 6f). Importantly, overexpression of the GSK3 $\beta$ K183R mutant significantly reduced mRNA expression of IL- 6 and TNF- $\alpha$ as well as c-Fos in a dose-dependent manner compared with that of the overexpressed wild-type GSK3 $\beta$ (Fig. 6g). In similar experiments, reconstitution of GSK3 $\beta$ but not GSK3 $\beta$ (K183R) mutant into Gsk3b $b^{-1}$ MEFs restored the poly I:C-induced IL-6, TNF- $\alpha$, IL-10 and c-Fos mRNA expression (Supplementary Fig. 16a,b) and GSK3 $\beta$ ubiquitination (Supplementary Fig. 16c).

Because GSK3 $\beta$ (1-120) lacking the K183 residue could bind to TRAF6 (Supplementary Fig. 13b), we tested whether GSK3 $\beta$ $(1-120)$ can be ubiquitinated by TRAF6. As expected, GSK3 $\beta$ (1-120) failed to be ubiquitinated by TRAF6 (Supplementary Fig. 17). Interestingly, GSK3 $\beta(1-120)$ could act as a dominantnegative mutant to inhibit poly I:C-induced mRNA expression of IL-6, TNF- $\alpha$ and c-Fos (Supplementary Fig. 18). 
a

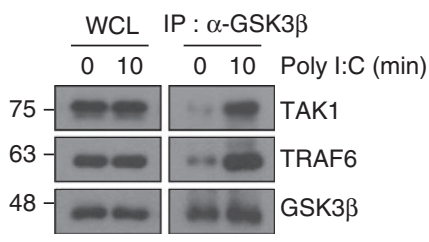

b

$$
\begin{aligned}
& ++++ \text { Flag-TRAF6 } \\
& -+-+ \text { Myc-TAK1 } \\
& -+++ \text { HA-GSK3 } \beta
\end{aligned}
$$

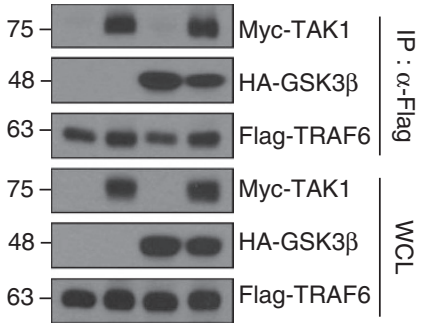

C

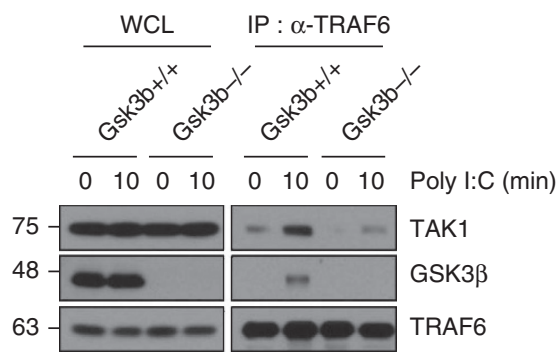

d

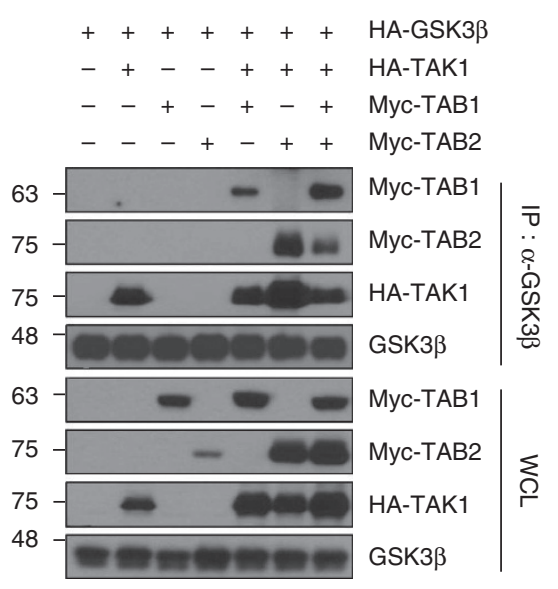

e

$$
\begin{aligned}
& +++ \text { Flag-TRAF6 } \\
& -++ \text { HA-TAK1 } \\
& -++ \text { Myc-TAB1 } \\
& -++ \text { Myc-TAB2 } \\
& -++ \text { HA-GSK3 } 3
\end{aligned}
$$

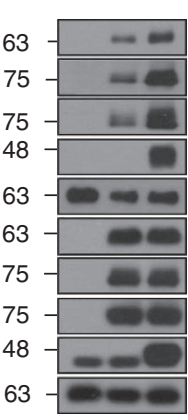

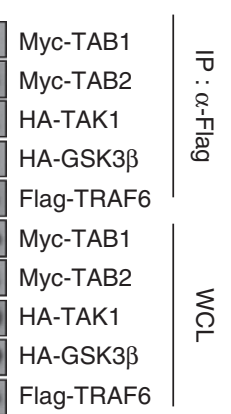

f

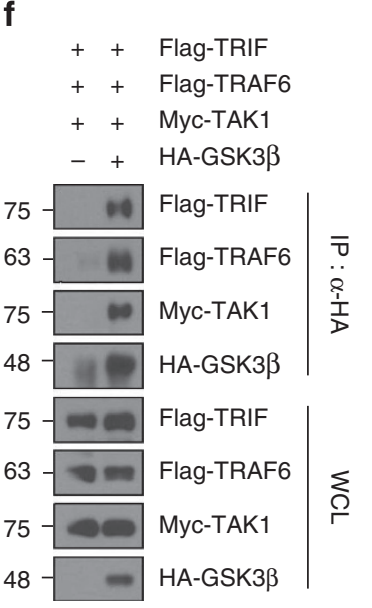

Figure 5 | GSK3 $\beta$ associates with TRAF6, TAK1 and TRIF. (a) BMDMs were stimulated with $10 \mu \mathrm{g} \mathrm{ml}{ }^{-1}$ poly l:C for 10 min and subjected to immunoprecipitation with an anti-GSK3 $\beta$ antibody. TRAF6 and TAK1 protein levels from whole-cell lysates (WCL) and TLR3 immunocomplexes (IP: $\alpha$-GSK3 $\beta$ ) were determined by western blotting. (b) GSK3 $\beta$ formed a ternary complex with TRAF6 and TAK1. HEK293T cells were transfected with the indicated combinations of expression plasmids. Co-imunoprecipitations were performed with anti-Flag antibody followed by western blotting with the indicated antibodies. The expression levels of the transfected plasmids were confirmed by western blot analysis of whole-cell lysates. (c) As in Fig. $4 \mathrm{~b}$, except that whole-cell lysates were immunoprecipitated with anti-TRAF6 antibody followed by western blotting as indicated. (d) GSK3 $\beta$ associated with TAB1 and TAB2 through TAK1. These experiments were performed as described in $\mathbf{b}$. (e) TRAF6 associated with TAB1, TAB2, TAK1 and GSK3B. The experiments were performed as described in $\mathbf{b}$. (f) GSK3 $\beta$ associates with TRIF, TRAF6 and TAK1. The experiments were performed as described in $\mathbf{b}$. Data are representative of two or three independent experiments.

GSK3 $\beta$ ubiquitination is required for interaction with TRAF6. In our earlier experiments, we showed that GSK3 $\beta$, TRAF6 and TAK1 bind to one another to form a ternary complex (Fig. 5b; Supplementary Fig. 11a,b) and that GSK3 $\beta$ associates with TRIF, TRAF6 and TAK1 as a complex (Fig. 5f). Therefore, we tested whether GSK3 $\beta$ ubiquitination influenced its interaction with TRIF, TRAF6 and TAK1. Overexpression of the GSK3 $\beta$ K183R mutant, which is defective in GSK3 $\beta$ ubiquitination by TRAF6, showed impaired association with TRAF6 compared with the association of wild-type GSK3 $\beta$ (Fig. 7a). However, the K183R mutation of GSK3 $\beta$ did not abrogate interactions with TRIF or TAK1 (Fig. 7b,c), suggesting that GSK3 $\beta$ ubiquitination by TRAF6 is essential for its interaction with TRAF6. Because either deficiency or a pharmacological inhibition of GSK3 $\beta$ impaired the poly I:C-triggered formation of the TLR3 signalling complex (Fig. 4), we tested whether the GSK3 $\beta$ K183R mutant produced similar effects. Forced expression of the GSK3 $\beta$ K183R mutant markedly decreased the association of GSK3 $\beta$ with TRIF, TRAF6 and TAK1 (Fig. 7d; Supplementary Fig. 19), as well as the formation of the TRIF-assembled signalling complex containing TRAF6, GSK3 $\beta$ and TAK1 (Fig. 7e). Together, our results show that TRAF6-mediated GSK3 $\beta$ ubiquitination is essential for TRAF6 interaction, thereby contributing to the formation of the TRIF-GSK3 $\beta$-TRAF6-TAK1 complex.

\section{Discussion}

In general, TLRs that recognize bacteria induce pro-inflammatory cytokines, whereas those TLRs that detect viruses trigger the IFN response ${ }^{2-4}$. These two responses depend on the engagement of the major two adaptor molecules, MyD88 (refs 28,29) and TRIF $^{8,9}$. All TLRs, with the exception of TLR3, signal through the MyD88-dependent pathway ${ }^{1,2}$, whereas TRIF-mediated signalling includes TLR3 (refs 6,7) and TLR4 (refs 35,36).GSK3 $\beta$ is a crucial regulator in the balance between pro- and anti-inflammatory cytokines in MyD88-dependent TLR signalling ${ }^{24}$, as well as viraltriggered RLR-mediated activation ${ }^{31}$. However, how GSK3 $\beta$ controls TLR3-mediated pro-inflammatory signalling mediated by TRIF, but not by MyD88, is still unknown. We therefore examined whether GSK3 $\beta$ is involved in pro-inflammatory cytokine production in TLR3 signalling and explored the molecuar basis of GSK3 $\beta$ 's role. We found that GSK3 $\beta$ selectively regulated the TLR3-mediated activation of ERK and p38 but not JNK or NF- $\kappa$ B. Notably, the ERK and p38 pathways were required for the induction of $\mathrm{c}$-Fos, which forms the AP1 complex ${ }^{2,14,38}$. We also found that GSK3 $\beta$ was incorporated into a TLR3-assembled multiprotein complex, and its signalling funtion was regulated by TRAF6-mediated ubiquitination. In the TLR3-assembled signalling complex, GSK3 $\beta$ undergoes polyubiquitination, which is dependent on the E3 ligase activity 
a

b

$0 \quad 10$ Poly I:C (min)

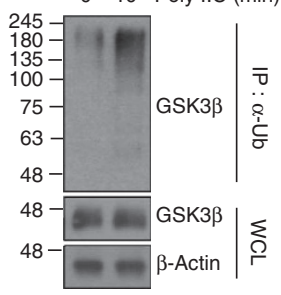

+++ HA-GSK3 3

-++ HA-Ub

$-\quad+$ Flag-TRAF6

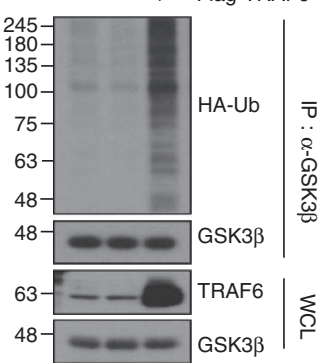

C

+++ HA-GSK3

-+++ HA-Ub

$-\quad+-$ Flag-TRAF6 (WT)

$-\quad+\quad+$ Flag-TRAF6 (C70A)

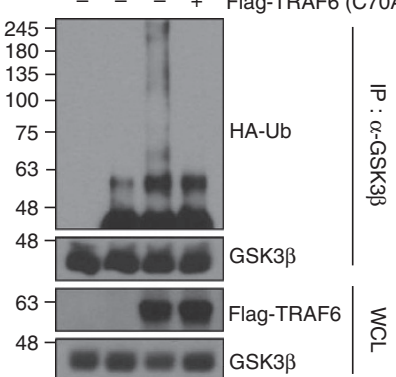

d

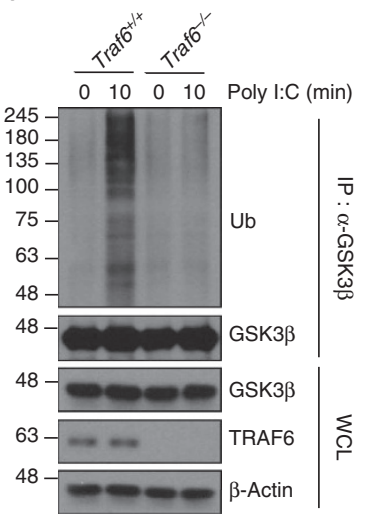

e

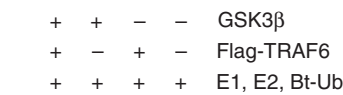

++++ E1, E2, Bt.

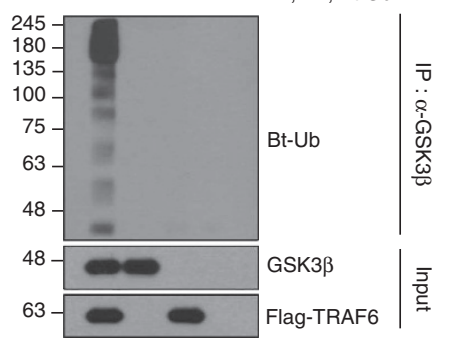

f

++++++++++ Ub

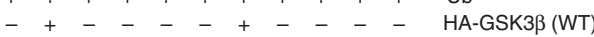

- $+\ldots+\ldots+\ldots$ - $+\ldots$ HA-GSK3B (K85R)

$-{ }_{-}+\ldots+\ldots+\ldots$ HA-GSK3B (K86R)

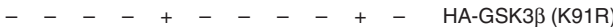

$-{ }_{-}-{ }_{-}+-{ }_{-}++$HA-GSK3ß (K183R)

$-+-\ldots++++$ Flag-TRAF6

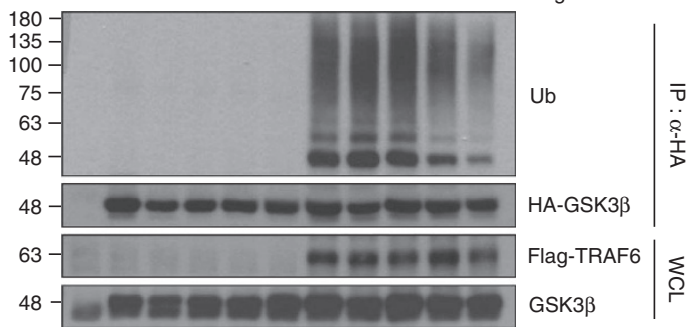

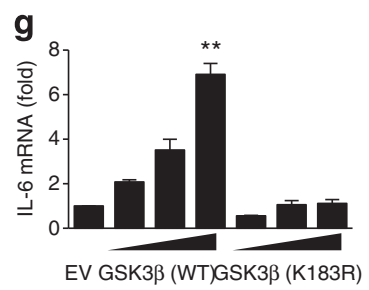
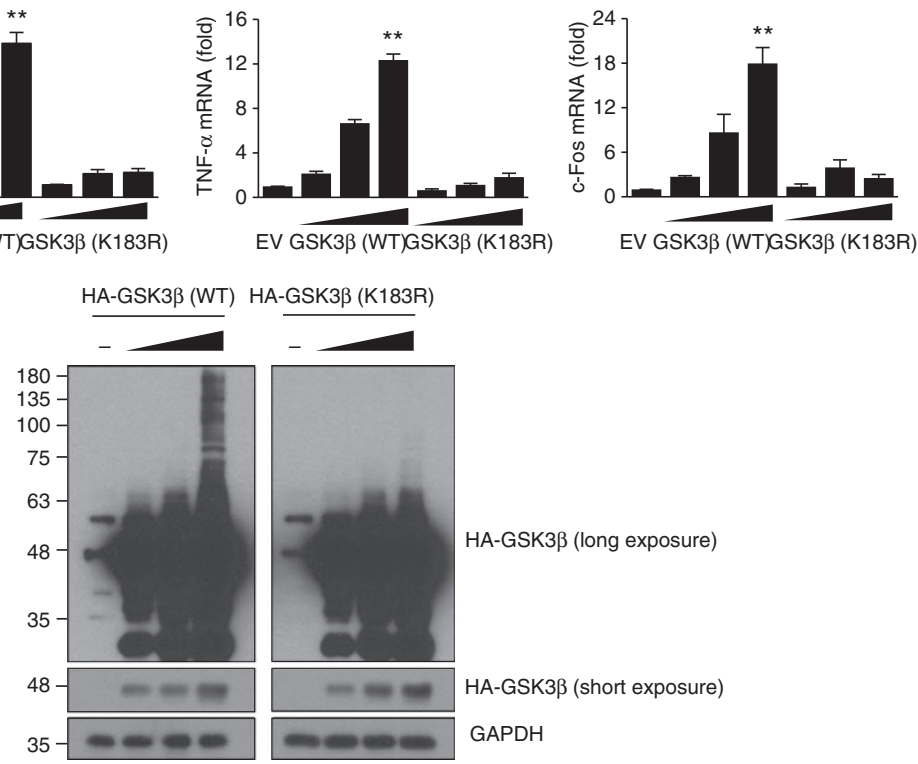

Figure 6 | TRAF6-mediated GSK3 $\beta$ ubiquitination at lysine 183 is critical for TLR3-dependent cytokine production. (a) BMDMs were stimulated with $10 \mu \mathrm{g} \mathrm{ml}^{-1}$ poly I:C for $10 \mathrm{~min}$ and subjected to immunoprecipitation with an anti-Ub antibody followed by western blotting with an anti-GSK3 $\beta$ antibody. (b) HEK293T cells transfected with HA-GSK3 $\beta$ and HA-Ub along with Flag-TRAF6 plasmids were subjected to immunoprecipitation with an anti-GSK3 $\beta$ antibody followed by western blotting with an anti-HA antibody. (c) HEK293T cells were transfected with HA-GSK3 $\beta$ and HA-Ub along with TRAF6 (WT) or TRAF6 (C7OA) plasmids. These experiments were performed as described in b. (d) Traf6 $6^{+/+}$and Traf6 $6^{-/-} 3 \mathrm{~T}_{3}$ cells stimulated with $10 \mu \mathrm{g}$ ml ${ }^{-1}$ poly I:C for 10 min were subjected to immunoprecipitation with an anti-GSK3 $\beta$ antibody followed by western blotting with an anti-Ub antibody. (e) GSK3 $\beta$ proteins were incubated with E1, E2 and biotinylated-Ub (Bt-Ub) in the presence or absence of Flag-TRAF6 proteins for in vitro ubiquitination of GSK3 3 . Ubiquitination of GSK3 $\beta$ was analysed by western blotting with streptavidin-HRP. (f) HEK293T cells transfected with Ub and Flag-TRAF6 along with HA-GSK3 $\beta$ WT or various HA-GSK3 $\beta$ mutants were subjected to immunoprecipitation with an anti-HA antibody followed by western blotting with an anti-Ub antibody. (g) HEK293-TLR3 cells were transiently transfected with GSK3 $\beta$ (WT) or GSK3 $\beta$ (K183R) plasmids. The levels of IL-6, TNF- $\alpha$ and c-Fos mRNA were determined by real-time PCR analysis (top). GSK3 $\beta$ expression levels were confirmed by western blotting with an anti-HA antibody (bottom). A longer exposure of the HA blot shows the presence of ubiquitin ladder. Data are presented as the mean \pm s.d. from at least three independent experiments. Statistical analyses were calculated using the Student's $t$-test $\left({ }^{\star \star} P<0.01\right)$. 
a

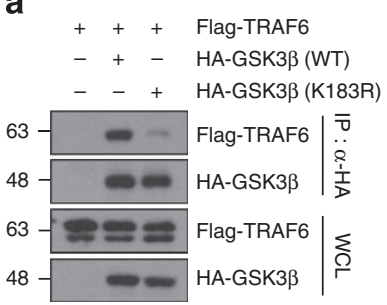

b

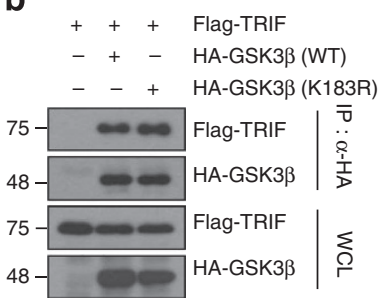

C

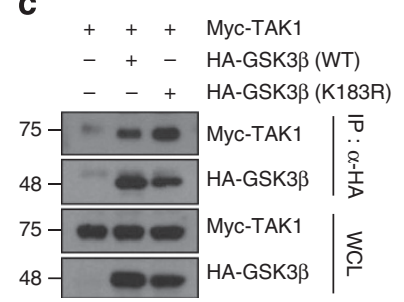

d

$$
\begin{array}{llll}
+ & + & + & \text { Flag-TRIF } \\
+ & + & + & \text { Flag-TRAF6 } \\
+ & + & + & \text { Myc-TAK1 } \\
- & + & - & \text { HA-GSK3 } \\
\text { (WT) } \\
- & - & + & \text { HA-GSK3 }(\text { K183R) }
\end{array}
$$

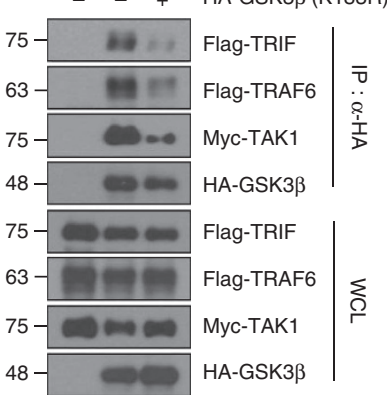

e

$$
\begin{array}{llll}
+ & + & + & \text { Flag-TRIF } \\
- & + & + & \text { GST-TRAF6 }
\end{array}
$$$$
-+++ \text { Myc-TAK1 }
$$$$
-\quad+\quad \text { HA-GSK3ß (WT) }
$$$$
-\quad+\quad+\text { HA-GSK3ß (K183R) }
$$

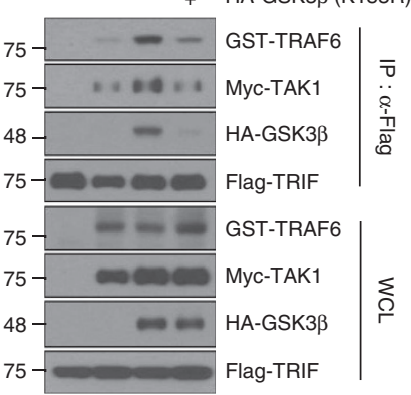

Figure 7 | Ubiquitination of GSK3 $\beta$ is required for the formation of a signalling complex containing TRIF, TRAF6, TAK1 and GSK3 3 . (a-c) The GSK3 $\beta$ K183R mutant abrogates the interaction of GSK3 $\beta$ with TRAF6 but not with TRIF or TAK1. HEK293T cells were transfected with the indicated combinations of expression plasmids. Co-imunoprecipitations were performed with an anti-HA antibody followed by western blotting with the indicated antibodies. The expression levels of the transfected plasmids were confirmed by western blotting of whole-cell lysates. (d,e) The GSK3 $\beta$ K183R mutation disrupts the TRIF-GSK3 $\beta$-TRAF6-TAK1 complex. HEK293T cells were transfected with the indicated combinations of expression plasmids. Co-imunoprecipitations were performed with anti-HA (d) or with anti-Flag (e) antibodies followed by western blotting with the indicated antibodies. The expression levels of the transfected plasmids were confirmed by western blotting of whole-cell lysates. Data are representative of two or three independent experiments.

of TRAF6, and thereby promotes the formation of the TRIFGSK3 $\beta$-TRAF6-TAK1 complex. Notably, a ubiquitinationdefective GSK3 $\beta$ mutant acts as a dominant-negative form of GSK3 $\beta$ regarding the induction of pro-inflammtory cytokines, as well as c-Fos. The present study establishes an important role for GSK3 $\beta$ in poly I:C-triggered inflammatory cytokine production and provides a mechanistic explanation for how the TRAF6GSK3 $\beta$ axis selectively regulates TLR3 signalling.

In addition, we found that GSK3 $\beta$ in TLR3 signalling had a positive role in regulating the production of TBK1-mediated type I IFN- $\beta$. Similar to our results, it was previously shown that GSK3 $\beta$ functions in RLR-mediated IFN- $\beta$ production by promoting TBK1 and IRF3 activation ${ }^{31}$, suggesting that the IFN- $\beta$ production triggered by TLR 3 and RLRs share a common pathway that converge upon TBK1, which is regulated by GSK3 $\beta$ through its kinase activity-independent mechanism. Among TRAF family members, TRAF3 positively regulates IRF3 and IFN- $\beta$ response through TRIF interaction ${ }^{10,52}$. Therefore, it is likely that the TRAF6-GSK3 $\beta$ axis controls MAPK signalling and c-Fos expression by TLR3, while the TRAF3-GSK3 $\beta$-TBK1 axis regulates IRF3 activation and IFN- $\beta$ induction. Indeed, we found that GSK3 $\beta$ interacts with TRAF3 (Supplementary Fig. 20a). Furthermore, GSK3 $\beta$ can form a complex containing TRIF, TRAF3 and TBK1 (Supplementary Fig. 20b).

Interestingly, GSK3 $\beta$ differentially regulates TLR-induced signalling. Our results have demonstrated that GSK3 $\beta$ regulates TLR3- and TLR4-mediated MAPK activation but is not required for TLR2 signalling ${ }^{53-55}$. Because TLR-mediated responses are controlled mainly by the MyD88-dependent pathway,28,29, which is used by all TLRs except TLR3, and the TRIFdependent pathway $8,9,30$, which is used by TLR3 and TLR4 (refs 35,36), we propose that GSK3 $\beta$ selectively regulates TRIFdependent MAPK activation. Our results also showed that GSK3 $\beta$ differentially regulates TLR3- and TLR4-mediated MAPKs signalling. GSK3 $\beta$ was required for ERK and p38 pathway activation downstream of TLR3, and for the JNK and p38 pathway activation downstream of TLR4. It is probable that endosomal TRIF signalling complexes downstream of TLR3 and TLR4 are not identical, and differences in their signalling potentials correlate with their ability to selelctively engage GSK $3 \beta$ and thereby dictate downstream MAPK activation. In TRIF-dependent TLR3 signalling, TRIF directly recruits TRAF6 and RIP1, which work cooperatively to activate TAK1, eventually leading to activation of NF- $\mathrm{B}$ and AP1 (refs 11,39,40). In the case of TLR4 stimulation, the initial step of TRIF signalling is mediated through an adaptor TRAM ${ }^{56,57}$. Internalized TLR4 recruits TRAF6 to the endosome via TRAM-TRIF ${ }^{58,59}$ Consequently, TRAF6 and RIP1 mediate the TRIF-induced activation of MAPKs and NF- $\kappa B$, respectively ${ }^{39,40}$. In this regard, the TRIF-assembled signalling complex of TLR3 or TLR4 formed in different ways, and this may have accounted for the differential regulation of GSK3 $\beta$ in MAPK activation. Although the exact mechanisms of the GSK3 $\beta$-mediated differential activation of MAPKs in the TLR3 and TLR4 signalling pathways require further investigations, our study suggests that GSK $3 \beta$ selectively regulates TRIF-dependent MAPK activation pathways.

Phosphorylation by GSK3 $\beta$ results in activation or inhibition of many its substrates $18,20,21$. Our results have demonstrated that the kinase activity of GSK3 $\beta$ is required for its ability to induce inflammatory cytokine production. These findings suggest that kinase activity of GSK3 $\beta$ in TLR3-TRIF signalling complex is 
involved in the cytokine production. Indeed, we found that Ser/Thr phosphorylation of TRAF6 was enhanced in control cells after poly I:C stimulation compared with that in the knockdown of GSK3 $\beta$ (Supplementary Fig. 21), suggesting that TRAF6 might be a candidate among GSK3 $\beta$ substrates. Alternatively, it has been recently reported that Bruton's tyrosine kinase is required for the production of inflammatory cytokines in TLR3-stimulated macrophages ${ }^{60}$, suggesting that Bruton's tyrosine kinase acts in TLR3/TRIF signalling. Further studies will be required for characterizing GSK3 $\beta$ substrate(s) in the TLR3-TRIF complex.

TRAF6, as an E3 ubiquitin ligase, is known to be a common signalling adaptor for cytokine production in response to various TLR ligands ${ }^{61,62}$. TRAF6 can ubiquinate itself on lysine 63 , and TRAF6 autoubiquitination in turn recruits mediators for the activation of downstream MAPKs and NF- $\mathrm{KB}$ signalling pathways $2,48,63$. TRAF6 mediates both MyD88-dependent and TRIF-dependent activation of NF-KB and AP1. In MyD88dependent and TRIF-dependent TLR signalling, ubiquitinated TRAF6 serves as a signalling scaffold to recruit TAK1 via TAB2 and TAB3 (refs 11,45,46,64). The TAK1 signalling complex, including TRAF6-TAB2-TAB3-TAB1-TAK1 is subsequently released into the cytosol, where TAK1 activates MAPK cascades ${ }^{43,46,64}$. Although TRAF6 appears to be a common factor employed by MyD88- and TRIF-dependnent signalling, it is probable that a specific signalling partner, substrate or other signalling protein(s) in each signalling complex is needed for signalling specificity or fine tuning of signalling. In this regard, we have now demonstrated that GSK3 $\beta$ underwent K63 chain ubiquitination. TRAF6 was found to be a direct E3 ligase for GSK3 $\beta$ and was essential for GSK3 $\beta$ ubiquitination, TRIFassembled signalling complex formation and pro-inflammtory cytokine production upon TLR3 stimulation. This mechanism involves the TLR3-induced assembly of a multiprotein complex containing TRIF, TRAF6, TAB1, TAB2, TAK1 and GSK3 $\beta$. Complex assembly resulted in TRAF6 autoubiquitination and activation, which led to K63-linked ubiquitination of GSK3 $\beta$. Ubiquitinated GSK3 $\beta$ promoted a multiprotein-assembled signalling complex, where TAK1 and its subordinate MAPKs are activated. Notably, during IL-1 and receptor activator of nuclear factor- $\mathrm{\kappa B}$ ligand signalling, TRAF6 autoubiquitination was dispensable for both interaction with and activation of the TAK1 signalling complex ${ }^{65}$. It has been suggested that TRAF6mediated K63-linked ubiquitination instead targets relevant protein substrates during activation 63,64 . Accordingly, we propose that TRAF6 mediates the K63-linked ubiquitination of GSK3 $\beta$, which would form a signalling complex sufficient to meet activation thresholds and/or to generate signalling specificity. It should be noted that TRAF6-mediated GSK3 $\beta$ ubiquitination proceeds through a two-stage mechanism. This mechanism involves an initial interaction prior to ubiquitination between TRAF6 and GSK3 $\beta$. The interaction of TRAF6 (through a coiledcoil TRAF-N domain and a conserved TRAF-C domain) with GSK3 $\beta$ (through a $\mathrm{N}$-terminal region spanning aa 1-120) may lead to K63-linked ubiquitination of GSK3 $\beta$ by E3 ligase activity of TRAF6. We thus propose that GSK $\beta$ is a novel TRAF6 substrate downstream of TLR3. The establishment of a regulatory role for GSK3 $\beta$ in TLR3-mediated signalling contributes to the elucidation of the complicated molecular mechanisms of inflammatory and antiviral responses.

\section{Methods}

Cell culture and transfection. MEFs from $G s k 3 b^{+/+}$and $G s k 3 b^{-/-}$mice were kindly provided by Dr J. Woodgett (Ontario Cancer Institute, Toronto, ON, Canada). 3T3 cell lines from Traf6 ${ }^{+/+}$and Traf6 ${ }^{-1-}$ mice were established in culture from E14.5 embryos using a standard 3T3 protocol ${ }^{66}$. HEK293-TLR3 stable cell lines were obtained from Dr I.H. Choi (Yonsei University College of Medicine,
Seoul, Republic of Korea). Cells were cultured in Dulbecco's modified Eagle's medium (DMEM, Hyclone, Logan, UT, USA) supplemented with $10 \%$ (v/v) fetal bovine serum (FBS, Hyclone), 100 units $^{-1}$ penicillin (Hyclone) and $100 \mu \mathrm{g} \mathrm{ml}^{-1}$ streptomycin (Hyclone). Murine BMDMs were obtained from the femurs of 8-10-week-old C57BL/6 male mice. Bone marrow cells were flushed out from the bone marrow cavity, suspended in DMEM supplemented with $20 \%$ (v/v) FBS. After 1 day, non-adherent cells were cultured in the presence of $10 \mathrm{ng} \mathrm{ml}^{-1}$ recombinant human macrophage colony-stimulating factor (R\&D Systems, Minneapolis, MN, USA). After 7 days, a homogeneous population of adherent macrophages was obtained. To generate the GSK3 $\beta$ knockdown RAW264.7 stable cell lines, non-targeting control and GSK $3 \beta$ shRNA $\left(5^{\prime}\right.$-CATGAAAGTTAGCA GAGATAA- $3^{\prime}$ ) plasmid constructs were purchased from Sigma (St Louis, MO, USA). RAW264.7 cells were transfected with either a non-targeting control or GSK $3 \beta$ shRNA plasmids using a Microporator MP-100 (Invitrogen, Carlsbad, CA, USA) and then selected in DMEM supplemented with $10 \%(\mathrm{v} / \mathrm{v})$ FBS containing $4 \mu \mathrm{g} \mathrm{ml}^{-1}$ puromycin (Sigma) for 2 weeks. HEK293T cells and HEK293-TLR3 cells were transfected with the indicated plasmids for $36 \mathrm{~h}$ using Lipofectamine 2000 (Invitrogen) according to the manufacturer's protocol. Non-targeting contro siRNA (sc-37007) and c-Fos siRNA (sc-29222) were purchased from Santa Cruz Biotechnology (Santa Cruz, CA, USA). GSK $3 \alpha$ siRNA (5'-AAAGCGTCAGTCGG GGCTATGTT- $\left.3^{\prime}\right)$ and GSK3 $\beta$ ( $5^{\prime}$-ACACGAAAGTGATTGGAAATT- $3^{\prime}$ ) siRNA were synthesized by Genolution (Seoul, Republic of Korea). For transient transfection of siRNA, cells were transfected with either a non-targeting control or target-specific siRNA duplexes for $36 \mathrm{~h}$ using a Lipofectamine RNAiMAX (Invitrogen) according to the manufacturer's protocol.

Reagents and antibodies. TLR3 agonist poly I:C, SB216763, BAY 11-7085, PD98059 and SB203580 were purchased from Sigma. Antibodies specific to phospho-GSK3 $\alpha / \beta$ (S21/S9, 9331, dilution 1:1,000), phospho-ERK (T202/Y204, 4370, dilution 1:1,000), phospho-JNK (T183/185, 9251, dilution 1:1,000), phosphop38 (T180/Y182, 9215, dilution 1:1,000), phospho-p65 (S536, 3031, dilution 1:1,000), GSK3 $\beta$ (9315, dilution 1:1,000) TAK1 (4505, dilution 1:1,000), ERK (4695, dilution $1: 1,000)$, JNK (9258, dilution $1: 1,000)$, p38 (8690, dilution 1:1,000), p65 (8242, dilution 1:1,000), ATF2 (9226, dilution 1:1,000) and c-Jun (9165, dilution 1:1,000) were purchased from Cell Signaling Technology (Beverly, MA, USA). Antibodies specific to TRAF6 (sc-7221, dilution 1:1,000), c-Fos (sc-7202, dilution 1:1,000), haemagglutinin (HA) (sc-7392, dilution 1:1,000), Myc (sc-40, dilution 1:1,000), glutathione S-transferase (GST) (sc-138, dilution 1:1,000), TBP (sc-204, dilution 1:1,000), ubiquitin (sc-8017, dilution 1:1,000), $\beta$-actin (sc-87778, dilution 1:1,000), $\alpha$-tubulin (sc-58666, dilution 1:1,000) and GAPDH (sc-87724, dilution 1:1,000) were from Santa Cruz Biotechnology. A GSK3 $\alpha / \beta$-specific antibody (44-610, dilution 1:5,000) was purchased from Invitrogen, and a RIP1specific antibody $(610459$, dilution 1:2,000) was purchased from BD Pharmingen (San Diego, CA, USA). A Flag-specific antibody was purchased from Sigma (F3156, dilution 1:5,000), and horseradish peroxidase (HRP)-conjugated secondary antibodies were obtained from Thermo Fisher Scientific (Waltham, MA, USA).

Plasmid constructs. Wild-type GSK3 $\beta$ plasmid was obtained from Dr J.K. Chung (Seoul National University, Seoul, Republic of Korea). To generate the various GSK3 $\beta$ (K85R, K86R, K91R and K183R) mutants, site-directed mutagenesis was performed by PCR. DNA fragments encoding the GSK $3 \beta$ and various deletion mutants were prepared by PCR and cloned into the pEGFP-N3 and pEBG expression vectors. The wild-type GSK $3 \alpha$ plasmid was provided by Dr J. Woodgett Wild-type and mutant TRAF6, TAB1 and TAB2 plasmids were described previously ${ }^{65}$. The wild-type TAK1 plasmid was a gift from Dr T. Ishitani (Kyushu University, Fukuoka, Japan). Wild-type ubiquitin plasmid was provided by Dr J.H. Seol (Seoul National University), and the wild-type TRIF plasmid was obtained from Dr W.S. Ryu (Yonsei University, Seoul, Republic of Korea).

Immunoprecipitation and western blot analysis. Cells were washed with cold PBS (Hyclone) and lysed with lysis buffer (50 mM Tris- $\mathrm{HCl}, \mathrm{pH} 8.0,150 \mathrm{mM} \mathrm{NaCl}$ $0.5 \%$ deoxycholate acid, $1 \% \mathrm{NP}-40$ ) containing phosphatase and protease inhibitors. For immunoprecipitation, lysates were incubated with the indicated primary antibodies at $4{ }^{\circ} \mathrm{C}$ for $16 \mathrm{~h}$, and were further incubated with protein A-agarose (Millipore, Billerica, MA, USA) at $4^{\circ} \mathrm{C}$ for $1 \mathrm{~h}$ with gentle shaking. After washing five times with lysis buffer, immunoprecipitated proteins were boiled with $2 \times$ SDS loading buffer, and separated on SDS-polyacrylamide gel electrophoresis (PAGE) and electrophoretically transferred to polyvinylidene difluoride membranes (Millipore). Membranes were blocked with 5\% BSA in Tris-buffered saline containing $0.1 \%$ Tween- 20 and were immunoreacted with the indicated primary antibodies and secondary antibodies conjugated to HRP. Images have been cropped for presentation. Full-size images of all western blots are provided in Supplementary Fig. 22.

Enzyme-linked immunosorbent assay. To measure mouse IL-6, TNF- $\alpha$ and IL-10 levels, BMDMs were preincubated with the indicated inhibitors and then stimulated with or without $10 \mu \mathrm{g} \mathrm{ml}^{-1}$ poly I:C for $20 \mathrm{~h}$. Cell culture supernatants were assessed using ELISA kits from R\&D Systems according to the manufacturer's instructions. 
Real-time PCR analysis. Total RNA was extracted from cells using the TRIzol reagent (Invitrogen) and reverse transcribed to complementary DNA using the Superscript cDNA synthesis kit (Invitrogen) following the manufacturer's instructions. Real-time PCR analysis was performed using the KAPA SYBR green FAST qPCR kit (Kapa Biosystems, Boston, MA, USA) on an ABI 7300 real-time PCR machine (Applied Biosystems, Foster City, CA). Samples were analysed in triplicate and normalized to $\beta$-actin mRNA expression. Primer sequences are listed in Supplementary Table 2

Cytosolic and nuclear fractionation. Cells were lysed with cytosolic extraction buffer (10 mM HEPES, pH 7.4, $10 \mathrm{mM} \mathrm{KCl,} 1.5 \mathrm{mM} \mathrm{MgCl}_{2}, 0.5 \mathrm{M}$ dithiothreitol, $0.05 \% \mathrm{NP}-40$ ) containing protease inhibitors. After centrifugation at 8,000 r.p.m. for $5 \mathrm{~min}$ at $4{ }^{\circ} \mathrm{C}$, supernatants were collected for the cytosolic fraction. Pellets were washed with cytosol extraction buffer and then lysed with nuclear extraction buffer (5 mM HEPES, pH 7.4, $300 \mathrm{mM} \mathrm{NaCl}, 1.5 \mathrm{mM} \mathrm{MgCl}_{2}, 0.2 \mathrm{mM}$ EDTA, 25\% glycerol) containing protease inhibitors. After incubation on ice for $30 \mathrm{~min}$, the nuclear fraction was obtained by centrifugation at 14,000 r.p.m. for $30 \mathrm{~min}$ at $4{ }^{\circ} \mathrm{C}$.

In vivo ubiquitination assay. The in vivo ubiquitination assay was performed as previously described ${ }^{67}$. Briefly, cells were lysed with SDS lysis buffer ( $50 \mathrm{mM}$ Tris- $\mathrm{HCl}, \mathrm{pH} 6.8,150 \mathrm{mM} \mathrm{NaCl}, 10 \%$ glycerol, $1 \%$ SDS) containing protease inhibitors. After boiling for $5 \mathrm{~min}$, lysates were diluted 10 -fold with dilution buffer (10 mM Tris-HCl, pH 8.0, $150 \mathrm{mM} \mathrm{NaCl}, 2 \mathrm{mM}$ EDTA, $1 \%$ Triton $\mathrm{X}-100$ ) containing protease inhibitors and incubated at $4{ }^{\circ} \mathrm{C}$ for $30 \mathrm{~min}$. After centrifugation at 20,000 r.p.m. for $30 \mathrm{~min}$ at $4{ }^{\circ} \mathrm{C}$, supernatants were subjected to immunoprecipitation with the indicated antibodies.

In vitro ubiquitination assay. The GSK $3 \beta$ protein was obtained from Invitrogen (cat \# PV3365) and the Flag-TRAF6 protein was purified as previously described ${ }^{68}$. Briefly, HEK293T cells transfected with Flag-TRAF6 for $48 \mathrm{~h}$ were lysed in a lysis buffer ( $10 \mathrm{mM}$ Tris- $\mathrm{HCl}, \mathrm{pH} 7.5,10 \mathrm{mM} \mathrm{NaCl}, 1.5 \mathrm{mM} \mathrm{MgCl}_{2}$ ) containing protease inhibitors and immunoprecipitated with an anti-Flag affinity gel (Sigma) for $16 \mathrm{~h}$ at $4^{\circ} \mathrm{C}$. Immunoprecipitates were washed and eluted with $300 \mu \mathrm{g} \mathrm{ml}^{-1}$ Flag peptide according to the manufacturer's instructions. The in vitro ubiquitination assay was performed as previously described with minor modifications ${ }^{67}$. Briefly, $5 \mathrm{nM}$ FlagTRAF6 and $1 \mu \mathrm{M}$ GSK3 $\beta$ protein were mixed with $100 \mathrm{nM}$ His-E1, $1 \mu \mathrm{M}$ His-E2 (Ubc13/Mms2) and $2.5 \mu \mathrm{M}$ Bt-Ub in $50 \mu \mathrm{M}$ ubiquitination reaction buffer from the ubiquitination kit (Enzo Life Sciences, Farmingdale, NY, USA) according to the manufacturer's instructions. Samples were subsequently immunoprecipitated with an anti-GSK3 $\beta$ antibody and separated on SDS-PAGE followed by streptavidin conjugated to HRP (Thermo Fisher Scientific).

GST pull-down assay. Cells were washed with cold $\mathrm{PBS}$ and lysed with lysis buffer (20 mM HEPES, pH 7.4, $150 \mathrm{mM} \mathrm{NaCl}, 150 \mathrm{mM} \mathrm{KCl}, 10 \mathrm{mM}$ EDTA, $10 \%$ glycerol, $1 \% \mathrm{NP}-40)$ containing phosphatase and protease inhibitors. Whole-cell lysates were incubated with glutathione-sepharose 4B (GE Healthcare, Piscataway, NJ, USA) at $4{ }^{\circ} \mathrm{C}$ for $3 \mathrm{~h}$ with gentle shaking. After washing three times with lysis buffer, proteins were boiled with $2 \times$ SDS loading buffer.

Native PAGE assay. For detection of IRF3 dimerization, Gsk $3 b^{+/+}$and Gsk $3 b^{-1-}$ MEFs stimulated with $10 \mu \mathrm{g} \mathrm{ml}^{-1}$ poly I:C for $1 \mathrm{~h}$ were lysed in a lysis buffer ( $50 \mathrm{mM}$ Tris- $\mathrm{HCl}, \mathrm{pH} 7.5,150 \mathrm{mM} \mathrm{NaCl}, 0.5 \% \mathrm{NP}-40$ ) containing protease inhibitors. After centrifugation at 20,000 r.p.m. for $10 \mathrm{~min}$, supernatants were mixed with $2 \times$ native sample buffer $(100 \mathrm{mM}$ Tris-Cl, $\mathrm{pH} 6.8,30 \%$ glycerol and $2 \%$ deoxycholate). Gels (7.5\%) (without SDS) were pre-run with $25 \mathrm{mM}$ Tris and $192 \mathrm{mM}$ glycine, $\mathrm{pH} 8.3$, with and without $1 \%$ deoxycholate in the cathode and anode chamber, respectively, for $1 \mathrm{~h}$ at $10 \mathrm{~mA}$ and $4^{\circ} \mathrm{C}$. Subsequently, samples were applied to the gel and electrophoresed for $\sim 1 \mathrm{~h}$ at $20 \mathrm{~mA}$ and $4{ }^{\circ} \mathrm{C}$ and transferred to polyvinylidene difluoride membranes (Millipore) for $1 \mathrm{~h}$ at $270 \mathrm{~mA}$ and $4{ }^{\circ} \mathrm{C}$.

Luciferase assay. RAW264.7 cells were transiently transfected with pGL3-Basic or pGL3-NF- $\kappa B-l u c$ along with pRL-TK-renilla luciferase plasmids. HEK293-null or HEK293-TLR3 cells were transiently transfected with pGL3-NF- $\kappa B-l u c$ and pRL-TK-renilla luciferase along with pEGFP-N3 or pEGFP-N3-GSK3 $\beta$ plasmids. After $24 \mathrm{~h}$ transfection, cells were stimulated with $10 \mu \mathrm{g} \mathrm{ml}^{-1}$ poly I:C for $4 \mathrm{~h}$ and luciferase activity was measured with the Dual-Luciferase Reporter Assay System (Promega, Madison, WI, USA) according to the manufacturer's instructions. Samples were analysed in triplicate and normalized to renilla luciferase activity.

Mapping of ubiquitination sites on GSK3及. For identification of ubiquitination sites on GSK3 $\beta$, HEK293T cells were transfected with HA-tagged GSK3 $\beta$ expression plasmids for $36 \mathrm{~h}$. Cells were lysed with lysis buffer $(20 \mathrm{mM}$ Tris- $\mathrm{HCl}, \mathrm{pH} 7.4$, $1 \%$ SDS), boiled for $5 \mathrm{~min}$, sonicated and then diluted 10 -fold with NP-40 lysis buffer (20 mM Tris-HCl, pH 7.4, 150 mM NaCl, 2 mM EDTA, 1\% NP-40). After centrifugation at $15,000 \mathrm{~g}$ for $15 \mathrm{~min}$, the lysates were incubated with $120 \mu \mathrm{l}$ of antiHA Agarose (Thermo scientific) at $4{ }^{\circ} \mathrm{C}$ overnight with rotation. The beads were washed four times with NP-40 lysis buffer and eluted with $2 \times$ SDS-PAGE sample buffer without a reducing agent. HA-GSK3 $\beta$ proteins eluted from the beads were subjected to SDS-PAGE followed by Coomassie Blue staining or western blot analysis. For MS analysis, gel bands from the Coomassie Blue stained-gel were excised and subjected to trypsin digestion and liquid chromatography-MS/MS MS/MS data were analysed using SEQUEST (Thermo Finnigan, San Jose, CA) software to identify ubiquitin modification with the GG or LRGG remnant tag on lysine residues of GSK3 $\beta$.

Statistical analysis. Data are presented as the mean \pm s.d. from at least three independent experiments. Statistical significance was determined using Student's $t$-test.

\section{References}

1. Medzhitov, R. \& Janeway, Jr. C. A. Innate immunity: the virtues of a nonclonal system of recognition. Cell 91, 295-298 (1997).

2. Akira, S. \& Takeda, K. Toll-like receptor signalling. Nat. Rev. Immunol. 4, 499-511 (2004).

3. Akira, S., Uematsu, S. \& Takeuchi, O. Pathogen recognition and innate immunity. Cell 124, 783-801 (2006).

4. Kawai, T. \& Akira, S. The role of pattern-recognition receptors in innate immunity: update on Toll-like receptors. Nat. Immunol. 11, 373-384 (2010).

5. Matsumoto, M. et al. Subcellular localization of Toll-like receptor 3 in human dendritic cells. J. Immunol. 171, 3154-3162 (2003).

6. Matsumoto, M. \& Seya, T. TLR3: interferon induction by double-stranded RNA including poly(I:C). Adv. Drug Deliv. Rev. 60, 805-812 (2008).

7. Perales-Linares, R. \& Navas-Martin, S. Toll-like receptor 3 in viral pathogenesis: friend or foe? Immunology 140, 153-167 (2013).

8. Yamamoto, M. et al. Role of adaptor TRIF in the MyD88-independent toll-like receptor signaling pathway. Science 301, 640-643 (2003).

9. Oshiumi, H., Matsumoto, M., Funami, K., Akazawa, T. \& Seya, T. TICAM-1, an adaptor molecule that participates in Toll-like receptor 3-mediated interferon-beta induction. Nat. Immunol. 4, 161-167 (2003).

10. Oganesyan, G. et al. Critical role of TRAF3 in the Toll-like receptor-dependent and -independent antiviral response. Nature 439, 208-221 (2006).

11. Sato, S. et al. Toll/IL-1 receptor domain-containing adaptor inducing IFN-beta (TRIF) associates with TNF receptor-associated factor 6 and TANK-binding kinase 1, and activates two distinct transcription factors, NF-kappa B and IFN-regulatory factor-3, in the Toll-like receptor signaling. J. Immunol. 171, 4304-4310 (2003).

12. Meylan, E. et al. RIP1 is an essential mediator of Toll-like receptor 3-induced NF-kappa B activation. Nat. Immunol. 5, 503-507 (2004).

13. Hiscott, J. Triggering the innate antiviral response through IRF-3 activation. J. Biol. Chem. 282, 15325-15329 (2007).

14. Karin, M., Liu, Z. J. \& Zandi, E. AP-1 function and regulation. Curr. Opin. Cell Biol. 9, 240-246 (1997).

15. Karin, M. \& Ben-Neriah, Y. Phosphorylation meets ubiquitination: the control of NF-[kappa]B activity. Annu. Rev. Immunol. 18, 621-663 (2000).

16. Embi, N., Rylatt, D. B. \& Cohen, P. Glycogen synthase kinase-3 from rabbit skeletal muscle. Separation from cyclic-AMP-dependent protein kinase and phosphorylase kinase. Eur. J. Biochem. 107, 519-527 (1980).

17. Woodgett, J. R. Molecular cloning and expression of glycogen synthase kinase-3/factor A. EMBO J. 9, 2431-2438 (1990).

18. Force, T. \& Woodgett, J. R. Unique and overlapping functions of GSK-3 isoforms in cell differentiation and proliferation and cardiovascular development. J. Biol. Chem. 284, 9643-9647 (2009).

19. Doble, B. W. \& Woodgett, J. R. GSK-3: tricks of the trade for a multi-tasking kinase. J. Cell Sci. 116, 1175-1186 (2003).

20. Jope, R. S. \& Johnson, G. V. The glamour and gloom of glycogen synthase kinase-3. Trends Biochem. Sci. 29, 95-102 (2004).

21. Rayasam, G. V., Tulasi, V. K., Sodhi, R., Davis, J. A. \& Ray, A. Glycogen synthase kinase 3: more than a namesake. Br. J. Pharmacol. 156, 885-898 (2009).

22. Beurel, E., Michalek, S. M. \& Jope, R. S. Innate and adaptive immune responses regulated by glycogen synthase kinase-3 (GSK3). Trends Immunol. 31, 24-31 (2010).

23. Wang, H., Brown, J. \& Martin, M. Glycogen synthase kinase 3: a point of convergence for the host inflammatory response. Cytokine 53, 130-140 (2011).

24. Martin, M., Rehani, K., Jope, R. S. \& Michalek, S. M. Toll-like receptormediated cytokine production is differentially regulated by glycogen synthase kinase 3. Nat. Immunol. 6, 777-784 (2005).

25 . $\mathrm{Hu}, \mathrm{X}$. et al. IFN-gamma suppresses IL-10 production and synergizes with TLR2 by regulating GSK3 and CREB/AP-1 proteins. Immunity 24, 563-574 (2006).

26. Rehani, K., Wang, H., Garcia, C. A., Kinane, D. F. \& Martin, M. Toll-like receptor-mediated production of IL-1Ra is negatively regulated by GSK3 via the MAPK ERK1/2. J. Immunol. 182, 547-553 (2009). 
27. Beurel, E. \& Jope, R. S. Glycogen synthase kinase-3 promotes the synergistic action of interferon-gamma on lipopolysaccharide-induced IL-6 production in RAW264.7 cells. Cell Signal. 21, 978-985 (2009).

28. Medzhitov, R. et al. MyD88 is an adaptor protein in the hToll/IL-1 receptor family signaling pathways. Mol. Cell 2, 253-258 (1998).

29. Kawai, T., Adachi, O., Ogawa, T., Takeda, K. \& Akira, S. Unresponsiveness of MyD88-deficient mice to endotoxin. Immunity 11, 115-122 (1999).

30. Hyun, J., Kanagavelu, S. \& Fukata, M. A unique host defense pathway: TRIF mediates both antiviral and antibacterial immune responses. Microbes Infect. 15, 1-10 (2013).

31. Lei, C. Q. et al. Glycogen synthase kinase $3 \beta$ regulates IRF3 transcription factor-mediated antiviral response via activation of the kinase TBK1. Immunity 33, 878-889 (2010).

32. Jiang, Z. et al. Poly(I-C)-induced Toll-like receptor 3 (TLR3)-mediated activation of NFkappa B and MAP kinase is through an interleukin-1 receptor-associated kinase (IRAK)-independent pathway employing the signaling components TLR3-TRAF6-TAK1-TAB2-PKR. J. Biol. Chem. 278, 16713-16719 (2003)

33. Jiang, Z., Mak, T. W., Sen, G. \& Li, X. Toll-like receptor 3-mediated activation of NF-kappaB and IRF3 diverges at Toll-IL-1 receptor domaincontaining adapter inducing IFN-beta. Proc. Natl Acad. Sci. USA 101, 3533-3538 (2004).

34. Hiscott, J. Convergence of the NF-kappaB and IRF pathways in the regulation of the innate antiviral response. Cytokine Growth Factor Rev. 18, 483-490 (2007).

35. Keck, S. et al. Absence of TRIF signaling in lipopolysaccharide-stimulated murine mast cells. J. Immunol. 186, 5478-5488 (2011).

36. Piao, W. et al. Recruitment of TLR adapter TRIF to TLR4 signaling complex is mediated by the second helical region of TRIF TIR domain. Proc. Natl Acad. Sci. USA 110, 19036-19041 (2013).

37. Zhong, B., Tien, P. \& Shu, H. B. Innate immune responses: crosstalk of signaling and regulation of gene transcription. Virology 352, 14-21 (2006)

38. Gauzzi, M. C., Del, Cornò, M. \& Gessani, S. Dissecting TLR3 signalling in dendritic cells. Immunobiology 215, 713-723 (2010).

39. Matsuzawa, A. et al. Essential cytoplasmic translocation of a cytokine receptor-assembled signaling complex. Science 321, 663-668 (2008).

40. Cusson-Hermance, N., Khurana, S., Lee, T. H., Fitzgerald, K. A. \& Kelliher, M. A. Rip1 mediates the Trif-dependent toll-like receptor 3- and 4-induced NF- $\{$ kappa $\}$ B activation but does not contribute to interferon regulatory factor 3 activation. J. Biol. Chem. 280, 36560-30566 (2005).

41. Yamaguchi, K. et al. Identification of a member of the MAPKKK family as a potential mediator of TGF-beta signal transduction. Science 270, 2008-2011 (1995).

42. Shim, J. H. et al. TAK1, but not TAB1 or TAB2, plays an essential role in multiple signaling pathways in vivo. Genes Dev. 19, 2668-2681 (2005).

43. Besse, A. et al. TAK1-dependent signaling requires functional interaction with TAB2/TAB3. J. Biol. Chem. 282, 3918-3928 (2007).

44. Shibuya, H. et al. TAB1: an activator of the TAK1 MAPKKK in TGF-beta signal transduction. Science 272, 1179-1182 (1996).

45. Takaesu, G. et al. TAB2, a novel adaptor protein, mediates activation of TAK1 MAPKKK by linking TAK1 to TRAF6 in the IL-1 signal transduction pathway. Mol. Cell 5, 649-658 (2000).

46. Morlon, A., Munnich, A. \& Smahi, A. TAB2, TRAF6 and TAK1 are involved in NF-kappaB activation induced by the TNF-receptor, Edar and its adaptator Edaradd. Hum. Mol. Genet. 14, 3751-3757 (2005).

47. Bradley, J. R. \& Pober, J. S. Tumor necrosis factor receptor-associated factors (TRAFs). Oncogene 20, 6482-6491 (2001).

48. Deng, L. et al. Activation of the IkappaB kinase complex by TRAF6 requires a dimeric ubiquitin-conjugating enzyme complex and a unique polyubiquitin chain. Cell 103, 351-361 (2000).

49. Ye, H. et al. Distinct molecular mechanism for initiating TRAF6 signalling. Nature 418, 443-447 (2002).

50. Fang, D. F. et al. NEDD4 ubiquitinates TRAF3 to promote CD40-mediated AKT activation. Nat. Commun. 5, 4513 (2014).

51. Perkins, D. J. et al. Reprogramming of murine macrophages through TLR2 confers viral resistance via TRAF3-mediated, enhanced interferon production. PLoS Pathog. 9, e1003479 (2013).

52. Tseng, P. H. et al. Different modes of ubiquitination of the adaptor TRAF3 selectively activate the expression of type I interferons and proinflammatory cytokines. Nat. Immunol. 11, 70-75 (2010).

53. Akira, S., Takeda, K. \& Kaisho, T. Toll-like receptors: critical proteins linking innate and acquired immunity. Nat. Immunol. 2, 675-680 (2001).
54. Kirschning, C. J. \& Schumann, R. R. TLR2: cellular sensor for microbial and endogenous molecular patterns. Curr. Top. Microbiol. Immunol. 270, 121-144 (2002).

55. Mele, T. \& Madrenas, J. TLR2 signalling: at the crossroads of commensalism, invasive infections and toxic shock syndrome by Staphylococcus aureus. Int. J Biochem. Cell Biol. 42, 1066-1071 (2010).

56. Bin, L. H., Xu, L. G. \& Shu, H. B. TIRP, a novel Toll/interleukin-1 receptor (TIR) domain-containing adapter protein involved in TIR signaling. J. Biol. Chem. 278, 24526-24532 (2003).

57. Yamamoto, M. et al. TRAM is specifically involved in the Toll-like receptor 4-mediated MyD88-independent signaling pathway. Nat. Immunol. 4, 1144-1150 (2003).

58. Yew, K. H., Carpenter, C., Duncan, R. S. \& Harrison, C. J. Human cytomegalovirus induces TLR4 signaling components in monocytes altering TIRAP, TRAM and downstream interferon-beta and TNF-alpha expression. PLoS ONE 7, e44500 (2012).

59. Fitzgerald, K. A. et al. LPS-TLR4 signaling to IRF-3/7 and NF-kappaB involves the toll adapters TRAM and TRIF. J. Exp. Med. 198, 1043-1055 (2003)

60. Lee, K. G. et al. Bruton's tyrosine kinase phosphorylates Toll-like receptor 3 to initiate antiviral response. Proc. Natl Acad. Sci. USA 109, 5791-5796 (2012).

61. Liu, Y. C. et al. Immunity by ubiquitylation: a reversible process of modification. Nat. Rev. Immunol. 5, 941-952 (2005).

62. Zinngrebe, J. et al. Ubiquitin in the immune system. EMBO Rep. 15, $28-45$ (2014).

63. Xie, P. TRAF molecules in cell signaling and in human diseases. J. Mol. Signal. 8, 7 (2013)

64. Adhikari, A., Xu, M. \& Chen, Z. J. Ubiquitin-mediated activation of TAK1 and IKK. Oncogene 26, 3214-3226 (2007).

65. Walsh, M. C., Kim, G. K., Maurizio, P. L., Molnar, E. E. \& Choi, Y. TRAF6 autoubiquitination-independent activation of the NFkappaB and MAPK pathways in response to IL-1 and RANKL. PLoS ONE 3, e4064 (2008).

66. Yoon, K. et al. TRAF6 deficiency promotes TNF-induced cell death through inactivation of GSK3beta. Cell Death Differ. 15, 730-738 (2008).

67. Wertz, I. E. et al. De-ubiquitination and ubiquitin ligase domains of A20 downregulate NF-kappaB signalling. Nature 430, 694-699 (2004).

68. Yang, W. L. et al. The E3 ligase TRAF6 regulates Akt ubiquitination and activation. Science 325, 1134-1138 (2009).

\section{Acknowledgements}

We thank Dr J. Woodgett (Ontario Cancer Institute) for MEFs and plasmids. We also thank Drs I.H. Choi (Yonsei University College of Medicine), J.K. Chung, J.H. Seol (Seoul National University), T. Ishitani (Kyushu University) and W.S. Ryu (Yonsei University) for sharing cell lines and plasmids. We thank Dr J. Kim (Ewha Womans University) for critical review of the manuscript. This work was supported by the National Research Foundation of Korea grant funded by the Korea Government (Ministry of Science, ICT \& Future Planning) (No. 2013R1A2A1A05005153; No. 2012R1A5A1048236; No.

2012M3A9C5048708). Y.C. was supported in part by grants from National Institutes of Health (AI064909 and AI08627).

\section{Author contributions}

R.K. and S.Y.L. designed experiments and analysed data. R.K. performed majority of the experiments. J.H.P. assisted with mRNA expression analysis. H.H. performed ubiquitination assays. Y.C. provided critical suggestions to this study. R.K. and S.Y.L. wrote the paper.

\section{Additional information}

Supplementary Information accompanies this paper at http://www.nature.com/ naturecommunications

Competing financial interests: The authors declare no competing financial interests.

Reprints and permission information is available online at http://npg.nature.com/ reprintsandpermissions/

How to cite this article: Ko, R. et al. Glycogen synthase kinase $3 \beta$ ubiquitination by TRAF6 regulates TLR3-mediated pro-inflammatory cytokine production. Nat. Commun. 6:6765 doi: 10.1038/ncomms7765 (2015).

This work is licensed under a Creative Commons Attribution 4.0 International License. The images or other third party material in this article are included in the article's Creative Commons license, unless indicated otherwise in the credit line; if the material is not included under the Creative Commons license, users will need to obtain permission from the license holder to reproduce the material To view a copy of this license, visit http://creativecommons.org/licenses/by/4.0/ 